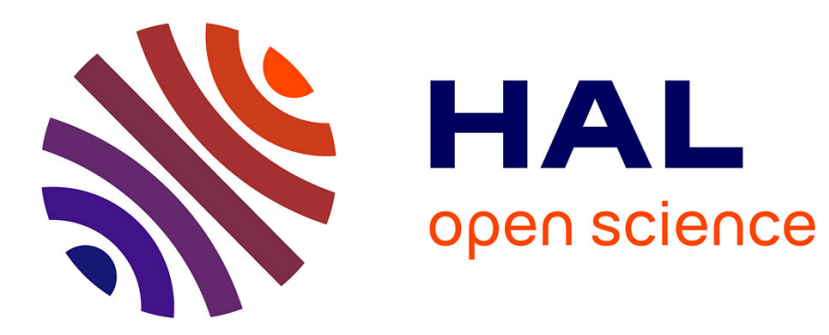

\title{
Convergence of a Discontinuous Galerkin scheme for the mixed time domain Maxwell's equations in dispersive media.
}

\author{
Claire Scheid, Stephane Lanteri
}

\section{- To cite this version:}

Claire Scheid, Stephane Lanteri. Convergence of a Discontinuous Galerkin scheme for the mixed time domain Maxwell's equations in dispersive media.. [Research Report] RR-7634, INRIA. 2011. inria-00597374

\section{HAL Id: inria-00597374 \\ https://hal.inria.fr/inria-00597374}

Submitted on 31 May 2011

HAL is a multi-disciplinary open access archive for the deposit and dissemination of scientific research documents, whether they are published or not. The documents may come from teaching and research institutions in France or abroad, or from public or private research centers.
L'archive ouverte pluridisciplinaire HAL, est destinée au dépôt et à la diffusion de documents scientifiques de niveau recherche, publiés ou non, émanant des établissements d'enseignement et de recherche français ou étrangers, des laboratoires publics ou privés. 


\title{
Convergence of a Discontinuous Galerkin scheme for the mixed time domain Maxwell's equations in dispersive media
}

\author{
Claire Scheid ${ }^{1}$ - Stéphane Lanteri ${ }^{2}$ \\ ${ }^{1}$ : INRIA Sophia Antipolis - Méditerranée research center, NACHOS project-team \\ and University of Nice - Sophia Antipolis \\ J.A. Dieudonné Mathematics Laboratory UMR CNRS 6621 \\ 2 : INRIA Sophia Antipolis - Méditerranée research center, NACHOS project-team \\ $\mathbf{N}^{\circ} 7634$
}

Mai 2011

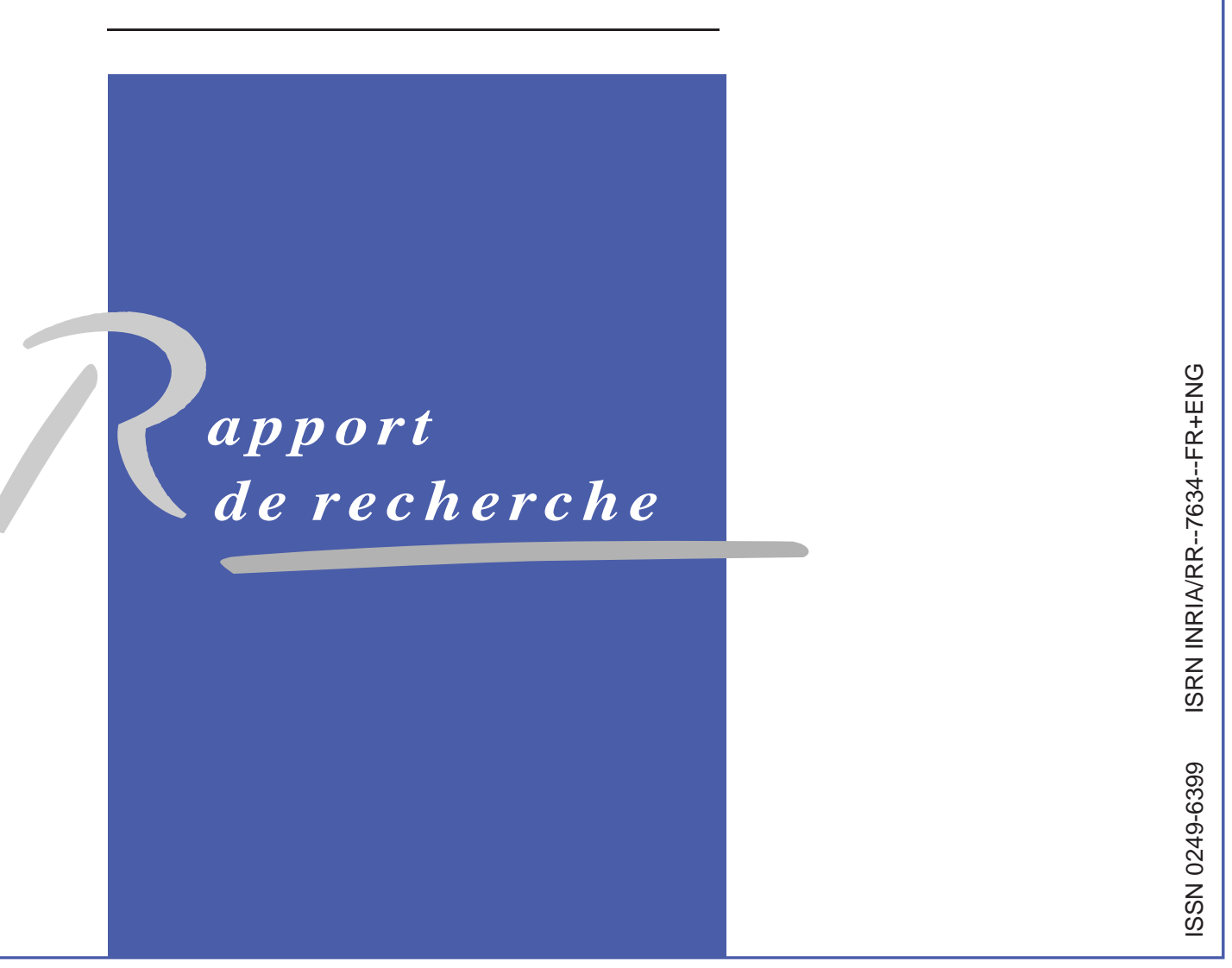





\title{
Convergence of a Discontinuous Galerkin scheme for the mixed time domain Maxwell's equations in dispersive media
}

\author{
Claire Scheid $^{1}$, Stéphane Lanteri ${ }^{2}$ \\ 1 : INRIA Sophia Antipolis - Méditerranée research center, NACHOs project-team \\ and University of Nice - Sophia Antipolis \\ J.A. Dieudonné Mathematics Laboratory UMR CNRS 6621 \\ 2 : INRIA Sophia Antipolis - Méditerranée research center, NACHOS project-team \\ Thème : Modélisation, simulation et analyse numérique \\ Équipe-Projet NACHOS \\ Rapport de recherche $\mathrm{n}^{\circ} 7634$ - Mai 2011 - 26] pages
}

\begin{abstract}
This study is concerned with the solution of the time domain Maxwell's equations in a dispersive propagation media by a Discontinuous Galerkin Time Domain (DGTD) method. The Debye model is used to describe the dispersive behaviour of the media. The resulting system of equations is solved using a centered flux discontinuous Galerkin formulation for the discretization in space and a second order leap-frog scheme for the integration in time. The numerical treatment of the dispersive model relies on an Auxiliary Differential Equation (ADE) approach similary to what is adopted in the Finite Difference Time Domain (FDTD) method. Stability estimates are derived through energy estimations and the convergence is proved for both the semi-discrete and the fully discrete case.
\end{abstract}

Key-words: Maxwell's equations, time domain, dispersive medium, discontinuous Galerkin method, convergence analysis. 


\section{Convergence d'un schéma Galerkin Discontinu pour les équations de Maxwell en formulation mixte et temporelle en milieu dispersif}

Résumé : On s'intéresse à la résolution numérique des équations de Maxwell en domaine temporel en milieu dispersif par une méthode Galerkin discontinue. Le caractère dispersif est ici pris en compte par le modèle de Debye. La méthode de résolution étudiée couple une formulation Galerkin discontinue à flux centré pour la discrétisation en espace et un schéma saute mouton du second ordre pour l'intégration en temps. Le traitement numérique du modèle dispersif repose sur une approche par équation différentielle auxiliaire à l'image de ce qui est réalisé dans la méthode de différences finies en domaine temporel. On étudie la stabilité du schéma résultant via des estimations d'énergie et prouvons la convergence des schémas semi-discrets et totalement discrets.

Mots-clés : Equations de Maxwell, domaine temporel, milieu dispersif, méthode Galerkin Discontinue, analyse de convergence. 


\section{Contents}

1 Introduction

2 The continuous problem 5

2.1 Formulation ............................. 5

2.2 Existence of a solution . . . . . . . . . . . . . . . . . . . . .

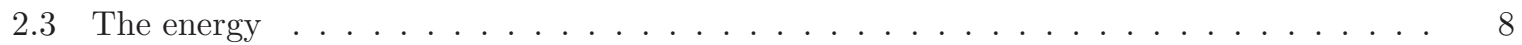

3 Semi-discretization by a DG method 9

3.1 Formulation ........................... 9

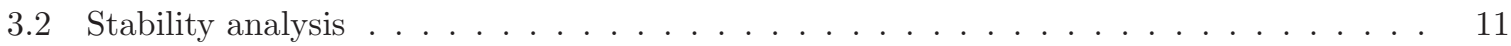

3.3 Convergence . . . . . . . . . . . . . . . . . . . . . . 11

3.3.1 Classical approximation results . . . . . . . . . . . . . . . . . 11

3.3 .2 Convergence result . . . . . . . . . . . . . . . . . . . . . . . . . . 12

4 Fully discrete study $\quad 14$

4.1 Formulation . . . . . . . . . . . . . . . . . . . . . . 14

4.2 Discrete energy . . . . . . . . . . . . . . . . . . . . . . . . . . . . . . . . . . . . . .

4.3 Convergence of the fully discrete scheme . . . . . . . . . . . . . . . 16

\begin{tabular}{llr}
5 & Numerical results & 20 \\
\hline
\end{tabular}

6 Conclusion $r 2$ 


\section{Introduction}

This study is concerned with the numerical modeling of the propagation of electromagnetic waves in dispersive media. Such propagation media are characterized by electromagnetic parameters (e.g. the electric permittivity) that depend on the frequency of the incident wave. There is a lot of practical problems that involve such propagation media and we are more particularly interested in situations for which one has to model the interaction of an electromagnetic wave with biological tissues. As a matter of fact, the numerical modeling of the propagation of electromagnetic waves through human tissues is at the heart of many biomedical applications such as breast imaging related to cancer tumor detection SKVH10, $\mathrm{KLG}^{+} 10$, the development of intelligent medical implants in the body [WSTI09] or microwave based hyperthermia to kill cancer cells [CBHV04, KKGU08. Finally, one can cite the electroporation technique which consists of applying nanopulses to the tissues, permitting to only affect intracellular membranes and then envisage treatments like electrochemiotherapy or gene transfer SKS $^{+} 92$, Tso91, $\mathrm{vCB}^{+} 05$, MvMM00, Ser05. In all these areas, there is a need for accurate and efficient numerical modeling techniques able to deal with the complex issues characterizing the associated propagation problems. Coming to the question of modeling electromagnetic waves leads to the study Maxwell's equations. The bibliography on Maxwell's equations is already well developed on both theoretical and numerical aspects (for a review see for example the book of P. Monk [Mon03], or the book [Bos98]). The problem of modeling electromagnetic waves propagating in human tissues however requires to taking into account the dispersive character of the propagation medium. Indeed human tissues contains a high percentage of water which gives them the properties to be dispersive. To be more precise, a dispersive medium is a medium where the speed of the propagating wave depends on the frequency.

The bibliography concerning the study of the propagation of electromagnetic waves in dispersive media is quite extended. For the numerical aspect of this problem, which is what this article is more concerned with, methodological developments have focused for quite a long time on the Finite Difference Time Domain (FDTD) method (see [Sul92, Lue90, KF90]). Numerical techniques based on the finite element method have also been investigated (see [Li06, LC06, Li07, LC08, LL08, Li11]). These studies deal with Nedelec elements and propose various proofs of convergence of semi-discrete scheme as well as in some of them fully discrete scheme with error estimates. Let us point out some of these articles. In [Li07, the mixed form of Maxwell's equations is considered, the study of a fully discrete scheme with first order accuracy is completed and some 2D numerical results are presented. In [LC06] and [LC08, the second order formulation of Maxwell's equation is considered and an example of fully discrete scheme analyzed. Finally in the very recent paper [Li11, the author considers the mixed formulation of Maxwell's equation and the dispersive character of the media is taken into account via an Auxiliary Differential Equation (ADE). The study focuses on a finite element formulation based on Nédélec elements and a leap-frog time integration scheme.

In the present work we develop such a numerical analysis for a discontinuous Galerkin discretization. Less work has been done on this topic, although a few works have been published very recently. One can cite LZC04, LCZ05, where no convergence proof is completed, and HL09, where error estimations are proved for the case of the second order formulation of Maxwell's equations and an interior penalty formulation for the case of cold plasma. The same interior penalty formulation is adopted in [HLY11] where furthermore some numerical tests are presented for another time scheme. In the recent article WXZ10, an error analysis is conducted for the semi-discrete case in a unified way for many dispersive media. The scheme is written and studied in its semi-discretized version, the fully-discrete scheme is described but not analyzed. Different dispersive media are presented, considering a locally divergence free discontinuous Galerkin methods. Some numerical results are also presented to validate the theoretical findings.

This work is intended as an attempt to go even further into the analysis of a fully discrete scheme for Maxwell's equations in dispersive media. In this article we present a complete numerical analysis study of the first order Maxwell's equations in dispersive media in a discontinuous Galerkin framework with 
an ADE approach. The mathematical model considered is the same than in [Li11 but its numerical treatment varies. The numerical scheme presented here is also different from the one presented in WXZ10, even if both approaches share common features. The starting point of our study is the nondissipative discontinuous Galerkin formulation presented and studied in [FLLP05 for the Maxwell's equations in non-dispersive media. We also include here an existence result in the continuous case and the analysis of the fully discrete case using a second order leap-frog scheme in time. Theoretical results are validated using an artificial test problem for which we can construct an analytical solution. In summary, the present study differs from previous works either from the point of view of the adopted mathematical model (mixed formulation and ADE technique), or by the numerical discretization used (non-dissipative DG method with centered fluxes).

This article is organized as follows. In section 2, we set up the equations of the model and the associated notations. The problem of existence of a solution is then studied using techniques from operator theory. Finally, we study the energy and prove stability. Section 3 is devoted to the study of the semi-discrete case. The discontinuous Galerkin formulation is described and then analysed via stability and convergence analysis in section 3. Section 4 contains the analysis of the fully discrete case. A CFL condition similar to FLLP05 is derived and convergence of the scheme then deduced. Finally section 4 gives some preliminary numerical results to validate the theory.

\section{The continuous problem}

In this section we state the initial and boundary value problem used to model the propagation of an electromagnetic wave in a dispersive medium. The physical behaviour of the medium is assumed to be described by the Debye model. We choose to use the ADE (Auxiliary Differential Equation) representation combined with the first order formulation of Maxwell's equations.

\section{$2.1 \quad$ Formulation}

Let $\Omega \subset \mathbb{R}^{3}$ be a bounded convex polyhedral domain. We denote by $\mathbf{n}$ the outward normal to $\partial \Omega$. Moreover, $\varepsilon_{0}$ (resp. $\mu_{0}$ ) is the electric (resp. magnetic) permittivity in free space, $\varepsilon_{\infty}\left(\right.$ resp. $\left.\mu_{\infty}\right)$ the relative electric permittivity of the medium at infinite frequency and $\sigma$ the conductivity of the medium. Furthermore:

$$
\begin{gathered}
\mathbf{L}^{2}(\Omega)=\left(L^{2}(\Omega)\right)^{3}, \\
\mathcal{H}(\Omega):=\mathcal{H}(\operatorname{curl}, \Omega) \times \mathcal{H}(\operatorname{curl}, \Omega) \times \mathbf{L}^{2}(\Omega),
\end{gathered}
$$

where $\mathcal{H}(\mathbf{c u r l}, \Omega)$ is the classical space of $\mathbf{L}^{2}$ fields with curl in $\mathbf{L}^{2}$, and:

$$
\begin{gathered}
\mathcal{L}(\Omega):=\left(\mathbf{L}^{2}(\Omega)\right)^{3}, \\
\mathbf{H}^{s}(\Omega)=\left(H^{s}(\Omega)\right)^{3} .
\end{gathered}
$$

The $L^{2}$ scalar product will be denoted by $\langle\cdot, \cdot\rangle$ and the associated norm $\|\cdot\|_{L^{2}(\Omega)}$. In a same way $\|\cdot\|_{X}$ will denote the canonical norm associated to the space $X .|\cdot|$ will denote the norm associated to the vector scalar product in $\mathbb{R}^{3}$ denoted by $\cdot$

We consider the time domain formulation of Maxwell's equation in $\Omega$. In a dispersive medium, the effect of the electric field is described by the electric displacement $\mathbf{D}$. One has:

$$
\mathbf{D}=\varepsilon_{0} \varepsilon_{\infty} \mathbf{E}+\mathbf{P}
$$

where $\mathbf{P}$ is called the polarization. The constitutive relation in a linear dispersive medium is defined in the frequency domain by:

$$
\hat{\mathbf{D}}(x, \omega)=\varepsilon_{0} \hat{\varepsilon}_{r}(x, \omega) \hat{\mathbf{E}}(x, \omega),
$$


where $\hat{\imath}$ denotes the Fourier transform of the corresponding function and $\varepsilon_{r}$ is called the relative permittivity. Its expression depends on the model used to describe the dispersive character of the medium (see (MPH06). In a time domain formulation we obtain:

$$
\mathbf{D}(x, t)=\varepsilon_{0} \varepsilon_{r}(x, t) \star \mathbf{E}(x, t) .
$$

For a Debye type dispersive medium, the frequency variation of relative permittivity is given by:

$$
\varepsilon_{r}(x, \omega)=\varepsilon_{\infty}+\frac{\varepsilon_{s}-\varepsilon_{\infty}}{1+j \omega \tau_{r}} .
$$

with $\varepsilon_{s}>\varepsilon_{\infty}$. This yields:

$$
\hat{\mathbf{D}}\left(1+j \omega \tau_{r}\right)=\varepsilon_{0} \varepsilon_{\infty}\left(1+j \omega \tau_{r}\right) \hat{\mathbf{E}}+\varepsilon_{0}\left(\varepsilon_{s}-\varepsilon_{\infty}\right) \hat{\mathbf{E}} .
$$

Taking the inverse Fourier transform, one obtains the Auxiliary Differential Equation (ADE) for D:

$$
\mathbf{D}+\tau_{r} \frac{\partial \mathbf{D}}{\partial t}=\varepsilon_{0} \varepsilon_{\infty} \tau_{r} \frac{\partial \mathbf{E}}{\partial t}+\varepsilon_{0} \varepsilon_{s} \mathbf{E} .
$$

and the ADE in terms of $\mathbf{P}$ writes:

$$
\mathbf{P}+\tau_{r} \frac{\partial \mathbf{P}}{\partial t}=\varepsilon_{0}\left(\varepsilon_{s}-\varepsilon_{\infty}\right) \mathbf{E} .
$$

We can now state Maxwell's equation in a Debye dispersive medium. Let $T>0$. The magnetic field $\mathbf{H}$, the electric field $\mathbf{E}$ and the polarization $\mathbf{P}$ verify the following system of equations in $[0, T]$ :

$$
\left\{\begin{array}{l}
\mu \frac{\partial \mathbf{H}}{\partial t}+\operatorname{curl} \mathbf{E}=0 \\
-\varepsilon_{0} \varepsilon_{\infty} \frac{\partial \mathbf{E}}{\partial t}+\operatorname{curl} \mathbf{H}=\frac{\partial \mathbf{P}}{\partial t}+\sigma \mathbf{E}, \\
\frac{\partial \mathbf{P}}{\partial t}=\frac{1}{\tau_{r}}\left[\varepsilon_{0}\left(\varepsilon_{s}-\varepsilon_{\infty}\right) \mathbf{E}-\mathbf{P}\right]
\end{array}\right.
$$

or equivalently:

$$
\left\{\begin{array}{l}
\mu \frac{\partial \mathbf{H}}{\partial t}+\operatorname{curl} \mathbf{E}=0, \\
\varepsilon_{0} \varepsilon_{\infty} \frac{\partial \mathbf{E}}{\partial t}-\operatorname{curl} \mathbf{H}=-\frac{1}{\tau_{r}}\left[\varepsilon_{0}\left(\varepsilon_{s}-\varepsilon_{\infty}\right) \mathbf{E}-\mathbf{P}\right]-\sigma \mathbf{E}, \\
\frac{\partial \mathbf{P}}{\partial t}=\frac{1}{\tau_{r}}\left[\varepsilon_{0}\left(\varepsilon_{s}-\varepsilon_{\infty}\right) \mathbf{E}-\mathbf{P}\right] .
\end{array}\right.
$$

We choose to work with metallic boundary conditions, i.e. $\mathbf{E} \times \mathbf{n}=0$ and initial conditions are given by $\mathbf{U}_{0}=\left(\mathbf{H}_{0}, \mathbf{E}_{0}, \mathbf{P}_{0}\right) \in \mathcal{H}(\Omega)$ such that $\mathbf{H}(0,)=.\mathbf{H}_{0}, \mathbf{E}(0,)=.\mathbf{E}_{0}, \mathbf{P}(0,)=.\mathbf{P}_{0}$.

Remark 2.1 The same analysis can be done with absorbing boundary conditions. The additional diffculties are given by some additional calculus like in [FLLP05].

We now state a weak formulation for the solution of system (11). Let:

$$
\beta:=\frac{\varepsilon_{0}}{\tau_{r}}\left(\varepsilon_{s}-\varepsilon_{\infty}\right) \quad \text { and } \quad \alpha:=\beta+\sigma .
$$


We seek the solution $\mathbf{U}=(\mathbf{H}, \mathbf{E}, \mathbf{P})$ in $\mathcal{C}([0, T], \mathcal{H}(\Omega))$ such that $\forall(\varphi, \psi, \phi) \in \mathcal{H}(\Omega)$ :

$$
\left\{\begin{array}{l}
\int_{\Omega} \mu \frac{\partial \mathbf{H}}{\partial t} \cdot \varphi+\int_{\Omega} \operatorname{curl} \mathbf{E} \cdot \varphi=0 \\
\int_{\Omega} \varepsilon_{0} \varepsilon_{\infty} \frac{\partial \mathbf{E}}{\partial t} \cdot \psi-\int_{\Omega} \operatorname{curl} \mathbf{H} \cdot \psi=\frac{1}{\tau_{r}} \int_{\Omega} \mathbf{P} \cdot \psi-\alpha \int_{\Omega} \mathbf{E} \cdot \psi \\
\int_{\Omega} \frac{\partial \mathbf{P}}{\partial t} \cdot \phi=-\frac{1}{\tau_{r}} \int_{\Omega} \mathbf{P} \cdot \phi+\beta \int_{\Omega} \mathbf{E} \cdot \phi
\end{array}\right.
$$

\subsection{Existence of a solution}

In order to prove the existence of a solution to the problem we consider, we will use operator theory (see CH90, Paz83 for details). One writes the equations (in a strong formulation) in a system form. If $\mathcal{U}=\left(U_{1}, U_{2}, U_{3}\right)$, with $U_{1}=\mathbf{H}, U_{2}=\mathbf{E}, U_{3}=\mathbf{P}$ :

$$
\left\{\begin{array}{l}
\Lambda \frac{d \mathcal{U}}{d t}=\mathcal{I}(\mathcal{U})+\mathcal{K}(\mathcal{U}) \\
\mathcal{U}(0)=U^{0}
\end{array}\right.
$$

with:

$$
\begin{gathered}
\mathcal{I}(\mathcal{U})=\left(-\operatorname{curl} U_{2}, \operatorname{curl} U_{1}, 0\right), \\
\mathcal{K}(\mathcal{U})=\left(0,-\alpha U_{2}+\frac{1}{\tau_{r}} U_{3}, \frac{1}{\tau_{r}} U_{2}-\frac{1}{\varepsilon_{0}\left(\varepsilon_{s}-\varepsilon_{\infty}\right) \tau_{r}} U_{3}\right),
\end{gathered}
$$

and:

$$
\Lambda=\left[\begin{array}{ccc}
\mu I_{3} & 0 & 0 \\
0 & \varepsilon_{0} \varepsilon_{\infty} I_{3} & 0 \\
0 & 0 & \frac{1}{\varepsilon_{0}\left(\varepsilon_{s}-\varepsilon_{\infty}\right)} I_{3}
\end{array}\right]
$$

The unbounded operator $\mathcal{I}$ is defined on $D(\mathcal{I})=\mathcal{H}(\operatorname{curl}, \Omega) \times \mathcal{H}_{0}(\operatorname{curl}, \Omega) \times \mathbf{L}^{2}(\Omega)$ which is dense in $\mathcal{L}(\Omega)$. Furthermore:

$$
\langle\mathcal{I}(\mathcal{U}), \mathcal{V}\rangle=-\langle\mathcal{U}, \mathcal{I}(\mathcal{V})\rangle, \quad \forall(\mathcal{U}, \mathcal{V}) \in D(\mathcal{I})^{2}
$$

This yields that:

$$
\mathcal{I}^{*}=-\mathcal{I}, \quad D\left(\mathcal{I}^{*}\right)=D(\mathcal{I}),
$$

and:

$$
\langle\mathcal{I}(\mathcal{U}), \mathcal{U}\rangle=0, \forall \mathcal{U} \in D(\mathcal{I})
$$

This implies that $\mathcal{I}$ is dissipative in $\mathcal{L}(\Omega)$.

The following lemma is central to prove our result of existence:

Lemma 2.2 Let $\nu>0$ be fixed. Then, the operator $\mathcal{I}-\nu \Lambda I$ is maximal-dissipative (for notations see [Paz83]) and is the infinitesimal generator of a $\mathcal{C}_{0}$ semigroup on $\mathcal{L}(\Omega)$.

Proof. For all $\mathcal{U} \in D(\mathcal{I}),\langle\mathcal{I}(\mathcal{U})-\nu \Lambda \mathcal{U}, \mathcal{U}\rangle=-\nu\left\|\Lambda^{\frac{1}{2}} \mathcal{U}\right\|_{\mathcal{L}(\Omega)}^{2} \leq-C\|\mathcal{U}\|_{\mathcal{L}(\Omega)}^{2}$. Same equality holds for $\mathcal{I}^{*}$. Thus $\mathcal{I}-\nu \Lambda I$ and $\mathcal{I}^{*}-\nu \Lambda I$ are also dissipative. Furthermore, since $\langle\mathcal{U}-(\mathcal{I}(\mathcal{U})-\nu \Lambda \mathcal{U}), \mathcal{U}\rangle=$ $\|\mathcal{U}\|_{\mathcal{L}(\Omega)}^{2}+\nu\left\|\Lambda^{\frac{1}{2}} \mathcal{U}\right\|_{\mathcal{L}(\Omega)}^{2}$, with the same equality for $\mathcal{I}^{*}$, one concludes that $\mathcal{I}-\nu \Lambda I$ and $\mathcal{I}^{*}-\nu \Lambda I$ are maximal dissipative. Results from semi-group theory as in [Paz83, allow to conclude.

Introducing $\mathcal{V}(t)$ such that $\mathcal{U}(t)=\exp (\nu t) \mathcal{V}(t), \mathcal{V}(t)$ satisfies the following equation:

$$
\left\{\begin{array}{l}
\Lambda \frac{d \mathcal{V}}{d t}-\mathcal{I}(\mathcal{V})+\nu \Lambda \mathcal{V}-\mathcal{K}(\mathcal{V})=0 \\
\mathcal{V}(0)=\mathcal{U}^{0}
\end{array}\right.
$$

RR $n^{\circ} 7634$ 
The operator $\mathcal{K}$ is linear and bounded on $\mathcal{L}(\Omega)$, with Theorem 1.1. from Paz83, Chapter 3, one deduces that $\mathcal{I}-\nu \Lambda+\mathcal{K}$ is also the infinitesimal generator of a $\mathcal{C}_{0}$ semigroup on $\mathcal{L}(\Omega)$. Then there exists a unique solution in the sense of Paz83. Therefore there exists a unique mild solution $\mathcal{V} \in \mathcal{C}^{0}([0, T], \mathcal{L}(\Omega))$. If $\mathcal{U} \in D(\mathcal{I})$ then it is a classical solution of the initial value problem.

One can precise the regularity in time for the solutions. We have that $\mathcal{W}=\frac{d \mathcal{V}}{d t}$ is solution of:

$$
\left\{\begin{array}{l}
\Lambda \frac{d \mathcal{W}}{d t}-\mathcal{I}(\mathcal{W})+\nu \mathcal{W}-\mathcal{K}(\mathcal{W})=0 \\
\mathcal{W}^{0}=\Lambda^{-1}(\mathcal{I}(\mathcal{W})-\nu \mathcal{W}+\mathcal{K}(\mathcal{W}))
\end{array}\right.
$$

If $\mathcal{U}^{0} \in D(\mathcal{I})$, then $\mathcal{W}^{0} \in \mathbf{L}^{2}(\Omega)$, and using the same arguments than before, one deduces that there exists a unique weak solution $\mathcal{W}$ to the previous equations with $\mathcal{W} \in \mathcal{C}^{0}([0, T], \mathcal{L}(\Omega))$. Then one can conclude that $\frac{d \mathcal{U}}{d t}=\frac{d}{d t}[\exp (\nu t) \mathcal{V}(t)]$ is a weak solution of the problem considered initially.

We can now turn to the corresponding solution $\mathcal{U}$ to conclude.

Theorem 2.3 If $\left(\mathbf{H}^{0}, \mathbf{E}^{0}, \mathbf{P}^{0}\right) \in D(\mathcal{I})$, then there exists a unique weak solution:

$$
(\mathbf{H}, \mathbf{E}, \mathbf{P}) \in \mathcal{C}^{0}([0, T], \mathcal{H}(\Omega)) \cap \mathcal{C}^{1}([0, T], \mathcal{L}(\Omega)),
$$

of the equation (2).

If more regularity is needed, one has to suppose more regularity on the initial conditions, especially on $(\mathbf{H}, \mathbf{E})$. The regularity for $\mathbf{P}$ is obtained even with $\mathbf{L}^{2}(\Omega)$ initial data. In the rest of the paper, we will for our needs suppose that furthermore $\left(\mathbf{H}^{0}, \mathbf{E}^{0}, \mathbf{P}^{0}\right) \in\left(H^{s+1}(\Omega) \times H^{s+1}(\Omega) \times L^{2}(\Omega)\right) \cap \mathcal{H}(\Omega)$, for $s>2$.

\subsection{The energy}

Let $\mathcal{E}$ define the energy of the system for $t \in[0, T]$ :

$$
\mathcal{E}(t)=\frac{1}{2}\left(\mu\|\mathbf{H}\|_{\mathbf{L}^{2}(\Omega)}^{2}(t)+\varepsilon_{0} \varepsilon_{\infty}\|\mathbf{E}\|_{\mathbf{L}^{2}(\Omega)}^{2}(t)+\frac{1}{\varepsilon_{0}\left(\varepsilon_{s}-\varepsilon_{\infty}\right)}\|\mathbf{P}\|_{\mathbf{L}^{2}(\Omega)}^{2}(t)\right) .
$$

We have the following result:

Proposition 2.4 The energy is decreasing, i.e. $\forall t \in[0, T]$ :

$$
\mathcal{E}(t) \leq \mathcal{E}(0) .
$$

Proof. Taking $\varphi=\mathbf{H}, \psi=\mathbf{E}$ et $\phi=\mathbf{P}$, as test functions in (2), one obtains:

$$
\left\{\begin{array}{l}
\mu\left\langle\frac{\partial \mathbf{H}}{\partial t}, \mathbf{H}\right\rangle+\langle\mathbf{c u r l} \mathbf{E}, \mathbf{H}\rangle=0 \\
\varepsilon_{0} \varepsilon_{\infty}\left\langle\frac{\partial \mathbf{E}}{\partial t}, \mathbf{E}\right\rangle-\langle\mathbf{H}, \mathbf{c u r l} \mathbf{E}\rangle=-\int_{\partial \Omega}(\mathbf{H} \times \mathbf{E}) \cdot n+\frac{1}{\tau_{r}}\langle\mathbf{P}, \mathbf{E}\rangle-\alpha\|\mathbf{E}\|_{\mathbf{L}^{2}(\Omega)}^{2} \\
\frac{1}{\varepsilon_{0}\left(\varepsilon_{s}-\varepsilon_{\infty}\right)}\left\langle\frac{\partial \mathbf{P}}{\partial t}, \mathbf{P}\right\rangle=-\frac{1}{\varepsilon_{0}\left(\varepsilon_{s}-\varepsilon_{\infty}\right) \tau_{r}}\|\mathbf{P}\|_{\mathbf{L}^{2}(\Omega)}^{2}+\frac{1}{\tau_{r}}\langle\mathbf{E}, \mathbf{P}\rangle
\end{array}\right.
$$

This yields:

$$
\begin{aligned}
\frac{1}{2}\left[\mu \frac{d}{d t}\|\mathbf{H}\|_{\mathbf{L}^{2}(\Omega)}^{2}+\varepsilon_{0} \varepsilon_{\infty} \frac{d}{d t}\|\mathbf{E}\|_{\mathbf{L}^{2}(\Omega)}^{2}+\frac{1}{\varepsilon_{0}\left(\varepsilon_{s}-\varepsilon_{\infty}\right)} \frac{d}{d t}\|\mathbf{P}\|_{\mathbf{L}^{2}(\Omega)}^{2}\right] & =-\frac{1}{\varepsilon_{0}\left(\varepsilon_{s}-\varepsilon_{\infty}\right) \tau_{r}}\|\mathbf{P}\|_{\mathbf{L}^{2}(\Omega)}^{2} \\
& +\frac{2}{\tau_{r}}\langle\mathbf{E}, \mathbf{P}\rangle-\alpha\|\mathbf{E}\|_{\mathbf{L}^{2}(\Omega)}^{2}
\end{aligned}
$$

RR $\mathrm{n}^{\circ} 7634$ 
so that:

$$
\frac{d}{d t} \mathcal{E}(t)=-\frac{1}{\tau_{r} \varepsilon_{0}\left(\varepsilon_{s}-\varepsilon_{\infty}\right)}\left\|\mathbf{P}-\varepsilon_{0}\left(\varepsilon_{s}-\varepsilon_{\infty}\right) \mathbf{E}\right\|_{\mathbf{L}^{2}(\Omega)}^{2}-\sigma\|\mathbf{E}\|_{\mathbf{L}^{2}(\Omega)}^{2}
$$

which gives the result.

\section{Semi-discretization by a DG method}

\subsection{Formulation}

We discretize the system (2) using a discontinuous Galerkin method formulated on simplicial meshes. We follow the approach of [FLLP05]. Let $\Omega_{h}$ be a discretization of the computational domain $\Omega$, using a triangulation $\mathcal{T}_{h}$ as follows: $\Omega_{h} \equiv \Omega, \mathcal{T}_{h}=\cup_{i=1}^{N_{\tau}} \tau_{i}$. We assume that the mesh is shape regular. An internal face is denoted by $a_{i k}=\tau_{i} \cap \tau_{k}$, for $i \neq k$ and $\mathbf{n}_{i k}$ is the unitary normal vector oriented from $\tau_{i}$ to $\tau_{k}$. For a given element $\tau_{i}, i \in\left[0, N_{\tau}\right]$, one denotes by $\mathcal{V}_{i}$ the set of the indices of all neighboring elements of $\tau_{i}$.

General setting. One seeks an approximation $\left(\mathbf{H}_{h}, \mathbf{E}_{h}, \mathbf{P}_{h}\right)$ of $(\mathbf{H}, \mathbf{E}, \mathbf{P})$ such that on each finite element $\tau_{i}, \mathbf{H}_{h}, \mathbf{E}_{h}, \mathbf{P}_{h}$ are polynomials whose degree can in all generality depend on the triangle $\tau_{i}$. For $i$ given, we denote by $d_{i}$ the number of degrees of freedom associated to the finite element $\tau_{i}$ and $\mathcal{P}_{i}$, the associated polynomial space. Moreover, $\left(\varphi_{i, j}\right)_{1 \leq j \leq d_{i}}$ defines a set of linearly independent functions such that $\mathcal{P}_{i}=\operatorname{Span}\left\{\varphi_{i, j}, 1 \leq j \leq d_{i}\right\}$. Then $\mathbf{H}_{h}, \mathbf{E}_{h}, \mathbf{P}_{h}$ are defined by: $\left(\mathbf{E}_{h}\right)_{/ \tau_{i}}=\mathbf{E}_{i},\left(\mathbf{H}_{h}\right)_{/ \tau_{i}}=\mathbf{H}_{i}$, $\left(\mathbf{P}_{h}\right)_{/ \tau_{i}}=\mathbf{P}_{i}$, where $\left(\mathbf{H}_{i}, \mathbf{E}_{i}, \mathbf{P}_{i}\right) \in \mathcal{P}_{i}^{9}$ and:

$$
\mathbf{V}_{h}=\left\{\mathbf{W}_{h} \in \mathbf{L}^{2}(\Omega) \quad \mid \quad\left(\mathbf{W}_{h}\right)_{/ \tau_{i}} \in\left(\mathcal{P}_{i}\right)^{3}, \quad \forall \tau_{i} \in \mathcal{T}_{h}\right\}
$$

the corresponding approximation space.

If $\mathbf{W}_{h} \in \mathbf{V}_{h}$ :

- $\mathbf{W}_{i}$ denotes its restriction to the element $\tau_{i}$.

- We define its average through any internal face $a_{i k}$, for given $(i, k)$ :

$$
\left\{\mathbf{W}_{h}\right\}_{i k}=\frac{\mathbf{W}_{i / a_{i k}}+\mathbf{W}_{k / a_{i k}}}{2},
$$

where $\mathbf{W}_{i / a_{i k}}$ denotes the restriction of $\mathbf{W}_{i}$ to the face $a_{i k}$.

- $\llbracket \mathbf{W}_{h} \rrbracket_{i k}$ stands for the tangential jump through any internal face $a_{i k}$ :

$$
\llbracket \mathbf{W}_{h} \rrbracket_{i k}=\left(\mathbf{W}_{k / a_{i k}}-\mathbf{W}_{i / a_{i k}}\right) \times \mathbf{n}_{i k} .
$$

In the approximation, we choose to use completely centered fluxes, i.e. the value of the field at an internal face is approximated by its average on this face. Finally $\mathcal{F}_{h}$ denotes the union of all the faces of the simplicial mesh $\mathcal{T}_{h}$ and $\mathcal{F}_{h}^{\text {int }}$ the union of all internal faces. A term $\int_{\mathcal{F}_{h}^{\text {int }}}$ will be understood as $\sum_{a_{i k} \in \mathcal{F}_{h}^{i n t}} \int_{a_{i k}}$.

In what follows, we consider the particular case where the discretization space is given by discontinuous piecewise polynomials of degree at most $k$ in each tetrahedron. In $\tau_{i}$, it is denoted by $\mathbb{P}_{k}\left(\tau_{i}\right)$. Then:

$$
\mathbf{V}_{h}=\left\{\mathbf{W}_{h} \in \mathbf{L}^{2}(\Omega) \quad \mid \quad\left(\mathbf{W}_{h}\right)_{/ \tau_{i}} \in\left(\mathbb{P}_{k}\left(\tau_{i}\right)\right)^{3}, \quad \forall \tau_{i} \in \mathcal{T}_{h}\right\}
$$


For what concern the treatment of the boundary condition on a metallic wall, for each of the corresponding boundary face (still denoted $a_{i k}$ ), we set $\mathbf{E}_{k / a_{i k}}=-\mathbf{E}_{i / a_{i k}}$, and $\mathbf{H}_{k / a_{i k}}=\mathbf{H}_{i / a_{i k}}$.

Taking the dot product of the equations of (1) by vectorial test functions $\varphi, \psi, \phi$ and integrating by part over $\tau_{i}$, one has:

$$
\left\{\begin{array}{l}
\mu \int_{\tau_{i}} \frac{\partial \mathbf{H}}{\partial t} \cdot \varphi+\int_{\tau_{i}} \mathbf{E} \cdot \operatorname{curl} \varphi=\int_{\partial \tau_{i}} \varphi \cdot(\mathbf{E} \times \mathbf{n}), \\
\varepsilon_{0} \varepsilon_{\infty} \int_{\tau_{i}} \frac{\partial \mathbf{E}}{\partial t} \cdot \psi-\int_{\tau_{i}} \mathbf{H} \cdot \mathbf{c u r l} \psi=-\int_{\partial \tau_{i}} \psi \cdot(\mathbf{H} \times \mathbf{n})+\frac{1}{\tau_{r}} \int_{\tau_{i}} \mathbf{P} \cdot \psi-\alpha \int_{\tau_{i}} \mathbf{E} \cdot \psi, \\
\int_{\tau_{i}} \frac{\partial \mathbf{P}}{\partial t} \cdot \phi=-\frac{1}{\tau_{r}} \int_{\tau_{i}} \mathbf{P} \cdot \phi+\beta \int_{\tau_{i}} \mathbf{E} \cdot \phi .
\end{array}\right.
$$

One seeks the semi-discrete solution $\mathbf{U}_{h}=\left(\mathbf{H}_{h}, \mathbf{E}_{h}, \mathbf{P}_{h}\right) \in \mathcal{C}^{1}\left(0, T, \mathbf{V}_{h}^{3}\right)$ as a solution of the following weak formulation, $\forall\left(\varphi_{h}, \psi_{h}, \phi_{h}\right) \in \mathbf{V}_{h}^{3}, \forall t \in[0, T], \forall i \in\left[0, N_{\tau}\right]$ :

$$
\left\{\begin{array}{r}
\mu \int_{\tau_{i}} \frac{\partial \mathbf{H}_{h}}{\partial t} \cdot \varphi_{h}+\int_{\tau_{i}} \mathbf{E}_{h} \cdot \operatorname{curl} \varphi_{h}=\sum_{k \in V_{i}} \int_{a_{i k}} \varphi_{h} \cdot\left(\left\{\mathbf{E}_{h}\right\}_{i k} \times \mathbf{n}\right), \\
\varepsilon_{0} \varepsilon_{\infty} \int_{\tau_{i}} \frac{\partial \mathbf{E}_{h}}{\partial t} \cdot \psi_{h}-\int_{\tau_{i}} \mathbf{H}_{h} \cdot \operatorname{curl} \psi_{h}=-\sum_{k \in V_{i}} \int_{a_{i k}} \psi_{h} \cdot\left(\left\{\mathbf{H}_{h}\right\}_{i k} \times \mathbf{n}\right) \\
\quad+\frac{1}{\tau_{r}} \int_{\tau_{i}} \mathbf{P}_{h} \cdot \psi_{h}-\alpha \int_{\tau_{i}} \mathbf{E}_{h} \cdot \psi_{h}, \\
\frac{1}{\varepsilon_{0}\left(\varepsilon_{s}-\varepsilon_{\infty}\right)} \int_{\tau_{i}} \frac{\partial \mathbf{P}_{h}}{\partial t} \cdot \phi_{h}=-\frac{1}{\varepsilon_{0}\left(\varepsilon_{s}-\varepsilon_{\infty}\right) \tau_{r}} \int_{\tau_{i}} \mathbf{P}_{h} \cdot \phi_{h}+\frac{1}{\tau_{r}} \int_{\tau_{i}} \mathbf{E}_{h} \cdot \phi_{h} .
\end{array}\right.
$$

We consider the following initial conditions:

$$
\mathbf{H}_{h}(0)=\pi_{h}\left(\mathbf{H}_{0}\right), \quad \mathbf{E}_{h}(0)=\pi_{h}\left(\mathbf{E}_{0}\right), \quad \mathbf{P}_{h}(0)=\pi_{h}\left(\mathbf{P}_{0}\right),
$$

where $\pi_{h}$ is the orthogonal $L^{2}$-projection onto $\mathbf{V}_{h}$.

Global formulation. Let $\mathbf{U}=(X, Y, Z)$ and $\mathbf{U}^{\prime}=\left(X^{\prime}, Y^{\prime}, Z^{\prime}\right)$ and let us define:

$$
\left\{\begin{aligned}
m\left(\mathbf{U}, \mathbf{U}^{\prime}\right) & =\mu \int_{\Omega} X \cdot X^{\prime}+\varepsilon_{0} \varepsilon_{\infty} \int_{\Omega} Y \cdot Y^{\prime}+\frac{1}{\varepsilon_{0}\left(\varepsilon_{s}-\varepsilon_{\infty}\right)} \int_{\Omega} Z \cdot Z^{\prime}, \\
a\left(\mathbf{U}, \mathbf{U}^{\prime}\right) & =\int_{\Omega}\left(X \cdot \operatorname{curl}_{h} Y^{\prime}-Y \operatorname{curl}_{h} X^{\prime}\right)-\alpha \int_{\Omega} Y \cdot Y^{\prime}+\frac{1}{\tau_{r}} \int_{\Omega} Y \cdot Z^{\prime} \\
& +\frac{1}{\tau_{r}} \int_{\Omega} Z \cdot Y^{\prime}-\frac{1}{\varepsilon_{0}\left(\varepsilon_{s}-\varepsilon_{\infty}\right) \tau_{r}} \int_{\Omega} Z \cdot Z^{\prime}, \\
b\left(\mathbf{U}, \mathbf{U}^{\prime}\right) & =\int_{\mathcal{F}_{h}^{\text {int }}}\{X\} \llbracket Y^{\prime} \rrbracket-\int_{\mathcal{F}_{h}^{\text {int }}}\{Y\} \llbracket X^{\prime} \rrbracket+\int_{\partial \Omega} Y^{\prime} \cdot(X \times n),
\end{aligned}\right.
$$

where $\operatorname{curl}_{h}$ is the piecewise curl operator, such that $\left(\operatorname{curl}_{h} V\right) / \tau_{i}=\operatorname{curl}\left(V_{/ \tau_{i}}\right)$.

Summing up all the contributions of (8) on each element, one obtains:

$$
m\left(\frac{\partial}{\partial t} \mathbf{U}_{h}, \mathbf{U}_{h}^{\prime}\right)=a\left(\mathbf{U}_{h}, \mathbf{U}_{h}^{\prime}\right)+b\left(\mathbf{U}_{h}, \mathbf{U}_{h}^{\prime}\right) \text { for all } \mathbf{U}_{h}^{\prime}=\left(\varphi_{h}, \psi_{h}, \phi_{h}\right) \in \mathbf{V}_{h}^{3} .
$$


Existence. One chooses a basis for the DG space and writes the equation in a matricial form. Then, solving these equations requires the inversion of the DG mass matrix and the resolution of a system of ordinary differential equations. This yields the existence of the semi-discrete solution.

Proposition 3.1 The weak formulation is consistent. In other words, if $\mathbf{U}$ is the exact solution then:

$$
m\left(\frac{\partial}{\partial t} \mathbf{U}, \mathbf{U}_{h}^{\prime}\right)=a\left(\mathbf{U}, \mathbf{U}_{h}^{\prime}\right)+b\left(\mathbf{U}, \mathbf{U}_{h}^{\prime}\right)
$$

for all $\mathbf{U}_{h}^{\prime}=\left(\varphi_{h}, \psi_{h}, \phi_{h}\right) \in \mathbf{V}_{h}^{3}$.

\subsection{Stability analysis}

Like in the continuous case, one defines the semi-discrete energy $\mathcal{E}_{h}$ :

$$
\mathcal{E}_{h}(t)=\frac{1}{2}\left(\mu\left\|\mathbf{H}_{h}\right\|_{\mathbf{L}^{2}(\Omega)}^{2}(t)+\varepsilon_{0} \varepsilon_{\infty}\left\|\mathbf{E}_{h}\right\|_{\mathbf{L}^{2}(\Omega)}^{2}(t)+\frac{1}{\varepsilon_{0}\left(\varepsilon_{s}-\varepsilon_{\infty}\right)}\left\|\mathbf{P}_{h}\right\|_{\mathbf{L}^{2}(\Omega)}^{2}(t)\right) .
$$

Proposition $3.2 \mathcal{E}_{h}$ is a decreasing function in time i.e. $\mathcal{E}_{h}(t) \leq \mathcal{E}_{h}(0)$.

Proof. Using the previously introduced bilinear forms, one has by (10):

$$
m\left(\frac{\partial}{\partial t} \mathbf{U}_{h}, \mathbf{U}_{h}\right)=a\left(\mathbf{U}_{h}, \mathbf{U}_{h}\right)+b\left(\mathbf{U}_{h}, \mathbf{U}_{h}\right)
$$

Simplifying the expressions one finds:

$$
\frac{d}{d t} \mathcal{E}_{h} \leq-\frac{1}{\tau_{r} \varepsilon_{0}\left(\varepsilon_{s}-\varepsilon_{\infty}\right)}\left\|\mathbf{P}-\varepsilon_{0}\left(\varepsilon_{s}-\varepsilon_{\infty}\right) \mathbf{E}\right\|_{\mathbf{L}^{2}(\Omega)}^{2}-\sigma\|\mathbf{E}\|_{\mathbf{L}^{2}(\Omega)}^{2} \leq 0 .
$$

Remark 3.3 This result follows directly from ideas of [FLLP05]. One just needs to consider in addition the contributions due to $\mathbf{E}$ and $\mathbf{P}$ which are of the same form than in the continuous case.

\subsection{Convergence}

In this section, we first recall some classical approximation results and then elaborate on the convergence of the introduced semi-discrete scheme.

\subsubsection{Classical approximation results}

Lemma 3.4 Let $\tau \in \mathcal{T}_{h}$ and $\Pi_{h}$ a linear continuous projector from $H^{s+1}(\tau)$ to $\mathbb{P}_{k}(\tau)$, for $s \geq 0$ and $k \geq 1$. Then if $u \in H^{s+1}(\tau)$ :

$$
\left|u-\Pi_{h}(u)\right|_{m, \tau} \leq C h_{\tau}^{\min (s, k)+1-m}\|u\|_{s+1, \tau}, \quad m=0,1
$$

and:

$$
\left\|u-\Pi_{h}(u)\right\|_{0, \partial \tau} \leq C h_{\tau}^{\min (s, k)+\frac{1}{2}}\|u\|_{s+1, \tau} .
$$

Lemma 3.5 (Inverse inequalities) Let $\tau \in \mathcal{T}_{h}$ and $k \geq 1$, then for all $p \in \mathbb{P}_{k}(\tau)$ :

$$
\begin{aligned}
\|p\|_{0, \partial \tau} & \leq C h_{\tau}^{-\frac{1}{2}}\|p\|_{0, \tau} \\
\|p\|_{1, \tau} & \leq C h_{\tau}^{-1}\|p\|_{0, \tau}
\end{aligned}
$$




\subsubsection{Convergence result}

We have the following result.

Theorem 3.6 Let $(\mathbf{H}, \mathbf{E}, \mathbf{P})$ be the exact solution of (1) and $\left(\mathbf{H}_{h}, \mathbf{E}_{h}, \mathbf{P}_{h}\right) \in \mathcal{C}^{1}\left([0, T], \mathbf{V}_{h}^{3}\right)$ be the semidiscrete solution of (7). If $(\mathbf{H}, \mathbf{E}, \mathbf{P}) \in \mathcal{C}^{0}\left([0, T], H^{s+1}(\Omega)^{9}\right)$ for $s \geq 0$, then there exists a constant $C>0$ independent of $h$ such that:

$$
\begin{gathered}
\max _{t \in[0, T]}\left(\left\|\pi_{h}(\mathbf{H})-\mathbf{H}_{h}\right\|_{\mathbf{L}^{2}(\Omega)}^{2}+\left\|\pi_{h}(\mathbf{E})-\mathbf{E}_{h}\right\|_{\mathbf{L}^{2}(\Omega)}^{2}+\left\|\pi_{h}(\mathbf{P})-\mathbf{P}_{h}\right\|_{\mathbf{L}^{2}(\Omega)}^{2}\right)^{\frac{1}{2}} \\
\leq C h^{\min (s, k)}\|(\mathbf{H}, \mathbf{E}, \mathbf{P})\|_{\mathcal{C}^{0}\left([0, T],\left(\mathbf{H}^{s+1}(\Omega)\right)^{3}\right)}
\end{gathered}
$$

where $\pi_{h}$ is the orthogonal $L^{2}$-projection on $\mathbf{V}_{h}$.

Proof. One needs an estimation of:

$$
\left\|\pi_{h}(\mathbf{E})-\mathbf{E}_{h}\right\|_{\mathbf{L}^{2}(\Omega)}^{2}+\left\|\pi_{h}(\mathbf{H})-\mathbf{H}_{h}\right\|_{\mathbf{L}^{2}(\Omega)}^{2}+\left\|\pi_{h}(\mathbf{P})-\mathbf{P}_{h}\right\|_{\mathbf{L}^{2}(\Omega)}^{2} .
$$

By abusing the notation, we will consider that $\pi_{h}(\mathbf{U})=\left(\pi_{h}(\mathbf{H}), \pi_{h}(\mathbf{E}), \pi_{h}(\mathbf{P})\right)$. If:

$$
\varepsilon(t)=\frac{1}{2} m\left(\pi_{h}(\mathbf{U})-\mathbf{U}_{h}, \pi_{h}(\mathbf{U})-\mathbf{U}_{h}\right),
$$

then there exists a constant $C>0$ such that:

$$
\varepsilon(t) \geq C\left[\left\|\pi_{h}(\mathbf{E})-\mathbf{E}_{h}\right\|_{\mathbf{L}^{2}(\Omega)}^{2}+\left\|\pi_{h}(\mathbf{H})-\mathbf{H}_{h}\right\|_{\mathbf{L}^{2}(\Omega)}^{2}+\left\|\pi_{h}(\mathbf{P})-\mathbf{P}_{h}\right\|_{\mathbf{L}^{2}(\Omega)}^{2}\right] .
$$

From the continuous equations and the semi-discrete ones, we can write:

$$
\left\{\begin{array}{l}
m\left(\frac{\partial}{\partial t} \mathbf{U}, \mathbf{U}_{h}^{\prime}\right)=a\left(\mathbf{U}, \mathbf{U}_{h}^{\prime}\right)+b\left(\mathbf{U}, \mathbf{U}_{h}^{\prime}\right) \\
m\left(\frac{\partial}{\partial t} \mathbf{U}_{h}, \mathbf{U}_{h}^{\prime}\right)=a\left(\mathbf{U}_{h}, \mathbf{U}_{h}^{\prime}\right)+b\left(\mathbf{U}_{h}, \mathbf{U}_{h}^{\prime}\right)
\end{array}\right.
$$

This gives:

$$
m\left(\frac{\partial}{\partial t}\left(\mathbf{U}-\mathbf{U}_{h}\right), \mathbf{U}_{h}^{\prime}\right)=a\left(\mathbf{U}-\mathbf{U}_{h}, \mathbf{U}_{h}^{\prime}\right)+b\left(\mathbf{U}-\mathbf{U}_{h}, \mathbf{U}_{h}^{\prime}\right) .
$$

Applying this relation to $\mathbf{U}_{h}^{\prime}=\pi_{h}(\mathbf{U})-\mathbf{U}_{h}$ yields:

$$
m\left(\frac{\partial}{\partial t}\left(\mathbf{U}-\mathbf{U}_{h}\right), \pi_{h}(\mathbf{U})-\mathbf{U}_{h}\right)=a\left(\mathbf{U}-\mathbf{U}_{h}, \pi_{h}(\mathbf{U})-\mathbf{U}_{h}\right)+b\left(\mathbf{U}-\mathbf{U}_{h}, \pi_{h}(\mathbf{U})-\mathbf{U}_{h}\right)=0
$$

Furthermore:

$$
\begin{aligned}
m\left(\frac{\partial}{\partial t} \pi_{h}(\mathbf{U})-\frac{\partial}{\partial t} \mathbf{U}_{h}, \pi_{h}(\mathbf{U})-\mathbf{U}_{h}\right) & =m\left(\frac{\partial}{\partial t} \pi_{h}(\mathbf{U})-\frac{\partial}{\partial t} \mathbf{U}+\frac{\partial}{\partial t} \mathbf{U}-\frac{\partial}{\partial t} \mathbf{U}_{h}, \pi_{h}(\mathbf{U})-\mathbf{U}_{h}\right) \\
& =m\left(\pi_{h}\left(\frac{\partial}{\partial t} \mathbf{U}\right)-\frac{\partial}{\partial t} \mathbf{U}, \pi_{h}(\mathbf{U})-\mathbf{U}_{h}\right) \\
& +m\left(\frac{\partial}{\partial t} \mathbf{U}-\frac{\partial}{\partial t} \mathbf{U}_{h}, \pi_{h}(\mathbf{U})-\mathbf{U}_{h}\right)
\end{aligned}
$$

Since $\pi_{h}$ is the orthogonal $L^{2}$-projection on $\mathbf{V}_{h}$ :

$$
m\left(\pi_{h}\left(\frac{\partial}{\partial t} \mathbf{U}\right)-\frac{\partial}{\partial t} \mathbf{U}, \pi_{h}(\mathbf{U})-\mathbf{U}_{h}\right)=0,
$$

RR $n^{\circ} 7634$ 
and

$$
\begin{aligned}
a\left(\pi_{h}(\mathbf{U})-\mathbf{U}, \pi_{h}(\mathbf{U})-\mathbf{U}_{h}\right) & =\left\langle\pi_{h}(\mathbf{E})-\mathbf{E}, \operatorname{curl}_{h}\left(\pi_{h}(\mathbf{H})-\mathbf{H}_{h}\right)\right\rangle \\
& -\left\langle\pi_{h}(\mathbf{H})-\mathbf{H}, \operatorname{curl}_{h}\left(\pi_{h}(\mathbf{E})-\mathbf{E}_{h}\right)\right\rangle \\
& -\alpha\left\langle\pi_{h}(\mathbf{E})-\mathbf{E}, \pi_{h}(\mathbf{E})-\mathbf{E}_{h}\right\rangle \\
& -\frac{1}{\varepsilon_{0}\left(\varepsilon_{s}-\varepsilon_{\infty}\right) \tau_{r}}\left\langle\pi_{h}(\mathbf{P})-\mathbf{P}, \pi_{h}(\mathbf{P})-\mathbf{P}_{h}\right\rangle \\
& +\frac{1}{\tau_{r}}\left\langle\pi_{h}(\mathbf{P})-\mathbf{P}, \pi_{h}(\mathbf{E})-\mathbf{E}_{h}\right\rangle \\
& +\frac{1}{\tau_{r}}\left\langle\pi_{h}(\mathbf{E})-\mathbf{E}, \pi_{h}(\mathbf{P})-\mathbf{P}_{h}\right\rangle=0,
\end{aligned}
$$

for the same reason. One then deduces from the last remark and (17):

$$
\begin{aligned}
m\left(\frac{\partial}{\partial t} \pi_{h}(\mathbf{U})-\frac{\partial}{\partial t} \mathbf{U}_{h}, \pi_{h}(\mathbf{U})-\mathbf{U}_{h}\right) & =m\left(\frac{\partial}{\partial t} \mathbf{U}-\frac{\partial}{\partial t} \mathbf{U}_{h}, \pi_{h}(\mathbf{U})-\mathbf{U}_{h}\right) \\
& =a\left(\mathbf{U}-\mathbf{U}_{h}, \pi_{h}(\mathbf{U})-\mathbf{U}_{h}\right)+b\left(\mathbf{U}-\mathbf{U}_{h}, \pi_{h}(\mathbf{U})-\mathbf{U}_{h}\right) \\
& =a\left(\mathbf{U}-\pi_{h}(\mathbf{U})+\pi_{h}(\mathbf{U})-\mathbf{U}_{h}, \pi_{h}(\mathbf{U})-\mathbf{U}_{h}\right) \\
& +b\left(\mathbf{U}-\mathbf{U}_{h}, \pi_{h}(\mathbf{U})-\mathbf{U}_{h}\right) \\
& =a\left(\pi_{h}(\mathbf{U})-\mathbf{U}_{h}, \pi_{h}(\mathbf{U})-\mathbf{U}_{h}\right)+b\left(\mathbf{U}-\mathbf{U}_{h}, \pi_{h}(\mathbf{U})-\mathbf{U}_{h}\right) \\
& =a\left(\pi_{h}(\mathbf{U})-\mathbf{U}_{h}, \pi_{h}(\mathbf{U})-\mathbf{U}_{h}\right)+b\left(\pi_{h}(\mathbf{U})-\mathbf{U}_{h}, \pi_{h}(\mathbf{U})-\mathbf{U}_{h}\right) \\
& +b\left(\mathbf{U}-\pi_{h}(\mathbf{U}), \pi_{h}(\mathbf{U})-\mathbf{U}_{h}\right) .
\end{aligned}
$$

One can quite easily show (as in previous sections and in [FLLP05]) that for any $\mathbf{U}_{h}^{\prime}=\left(X_{h}^{\prime}, Y_{h}^{\prime}, Z_{h}^{\prime}\right)$ in $\left(\mathbf{V}_{h}\right)^{3}$ :

$$
a\left(\mathbf{U}_{h}^{\prime}, \mathbf{U}_{h}^{\prime}\right)+b\left(\mathbf{U}_{h}^{\prime}, \mathbf{U}_{h}^{\prime}\right)=-\alpha\left\|Y_{h}^{\prime}\right\|_{\mathbf{L}^{2}(\Omega)}^{2}+\frac{2}{\tau_{r}}\left\langle Y_{h}^{\prime}, Z_{h}^{\prime}\right\rangle-\frac{1}{\varepsilon_{0}\left(\varepsilon_{s}-\varepsilon_{\infty}\right) \tau_{r}}\left\|Z_{h}^{\prime}\right\|_{\mathbf{L}^{2}(\Omega)}^{2} .
$$

Same argument than in previous proofs, gives that

$$
a\left(\mathbf{U}_{h}^{\prime}, \mathbf{U}_{h}^{\prime}\right)+b\left(\mathbf{U}_{h}^{\prime}, \mathbf{U}_{h}^{\prime}\right) \leq 0 .
$$

Then, since:

$$
\varepsilon(t)=\frac{1}{2} \int_{0}^{t} m\left(\partial_{s}\left(\pi_{h}(\mathbf{U})-\mathbf{U}_{h}\right), \pi_{h}(\mathbf{U})-\mathbf{U}_{h}\right) d s,
$$

one can write:

$$
\varepsilon(t) \leq \frac{1}{2} \int_{0}^{t} b\left(\mathbf{U}-\pi_{h}(\mathbf{U}), \pi_{h}(\mathbf{U})-\mathbf{U}_{h}\right)
$$

It only remains to estimate the last term involving $b$. We will refer to FLLP05, since this follows exactly their proof of theorem 3.5. on page 1163. One obtains:

$$
\begin{gathered}
b\left(\pi_{h}(\mathbf{U})-\mathbf{U}, \pi_{h}(\mathbf{U})-\mathbf{U}_{h}\right) \\
\leq C h^{\min (s, k)}\left(\left\|\pi_{h}(\mathbf{E})-\mathbf{E}_{h}\right\|_{\mathbf{L}^{2}(\Omega)}^{2}+\left\|\pi_{h}(\mathbf{H})-\mathbf{H}_{h}\right\|_{\mathbf{L}^{2}(\Omega)}^{2}\right)^{\frac{1}{2}}\|(\mathbf{H}, \mathbf{E})\|_{\left(\mathbf{H}^{s+1}(\Omega)\right)^{2} .}
\end{gathered}
$$


Let:

$$
\gamma(t)=\left\|\pi_{h}(\mathbf{H})-\mathbf{H}_{h}\right\|_{\mathbf{L}^{2}(\Omega)}^{2}+\left\|\pi_{h}(\mathbf{E})-\mathbf{E}_{h}\right\|_{\mathbf{L}^{2}(\Omega)}^{2}+\left\|\pi_{h}(\mathbf{P})-\mathbf{P}_{h}\right\|_{\mathbf{L}^{2}(\Omega)}^{2},
$$

and:

$$
\delta=\max _{t \in[0, T]} \gamma(t)^{\frac{1}{2}}
$$

$\delta$ is well defined due to the regularity hypotheses on the solutions $(\mathbf{H}, \mathbf{E}, \mathbf{P})$ and $\left(\mathbf{H}_{h}, \mathbf{E}_{h}, \mathbf{P}_{h}\right)$. Then

$$
\varepsilon(t) \leq C h^{\min (s, k)} \int_{0}^{t} \gamma(u)^{\frac{1}{2}}\|(\mathbf{H}, \mathbf{E})\|_{\left(\mathbf{H}^{s+1}(\Omega)\right)^{2}} d u
$$

One deduces for all $t \in[0, T]$ :

$$
\frac{\gamma(t)}{\delta} \leq C h^{\min (s, k)}\|(\mathbf{H}, \mathbf{E})\|_{\mathcal{C}^{0}\left([0, T],\left(\mathbf{H}^{s+1}(\Omega)\right)^{2}\right)},
$$

which leads to the result.

Error estimation is then easily deduced from (16). Indeed one makes use of:

$$
\mathbf{U}-\mathbf{U}_{h}=\mathbf{U}-\pi_{h}(\mathbf{U})+\pi_{h}(\mathbf{U})-\mathbf{U}_{h}
$$

and the classical approximation results recalled previously. The approximation error then keeps the same order.

Corollary 3.7 Under the same assumptions than those of theorem [3.6, there exists a constant $C>0$ such that:

$$
\begin{gathered}
\left(\left\|\mathbf{H}-\mathbf{H}_{h}\right\|_{\mathcal{C}^{0}\left([0, T], \mathbf{L}^{2}(\Omega)\right)}^{2}+\left\|\mathbf{E}-\mathbf{E}_{h}\right\|_{\mathcal{C}^{0}\left([0, T], \mathbf{L}^{2}(\Omega)\right)}^{2}+\left\|\mathbf{P}-\mathbf{P}_{h}\right\|_{\mathcal{C}^{0}\left([0, T], \mathbf{L}^{2}(\Omega)\right)}^{2}\right)^{\frac{1}{2}} \\
\leq C h^{\min (s, k)}\|(\mathbf{H}, \mathbf{E}, \mathbf{P})\|_{\mathcal{C}^{0}\left([0, T],\left(\mathbf{H}^{s+1}(\Omega)\right)^{3}\right)} .
\end{gathered}
$$

\section{Fully discrete study}

The semi-discrete scheme is discretized in time using the leap-frog or Störmer Verlet scheme (for a formulation see [HLW06]). This scheme has the advantages of being explicit. On the other hand, the price to pay is that a CFL condition has to be satisfied.

\subsection{Formulation}

We consider a fixed time step $\Delta t$. The unknowns for the electric field and the polarization are approximated at integer time station $t^{n}=n \Delta t$ with notation $\mathbf{E}^{n}$ and $\mathbf{P}^{n}$. The unknowns for the magnetic field are approximated at half-integer time station $t^{n+\frac{1}{2}}=\left(n+\frac{1}{2}\right) \Delta t$ with notation $\mathbf{H}^{n+\frac{1}{2}}$. Let $\mathbf{E}^{\left[n+\frac{1}{2}\right]}=\frac{\mathbf{E}^{n}+\mathbf{E}^{n+1}}{2}$ and $\mathbf{P}^{\left[n+\frac{1}{2}\right]}=\frac{\mathbf{P}^{n}+\mathbf{P}^{n+1}}{2}$. 
One seeks $\left(\mathbf{H}_{h}^{n+\frac{3}{2}}, \mathbf{E}_{h}^{n+1}, \mathbf{P}_{h}^{n+1}\right)$ such that $\forall \varphi_{h} \in \mathbf{V}_{h}, \forall \psi_{h} \in \mathbf{V}_{h}, \forall \phi_{h} \in \mathbf{V}_{h}$ :

$$
\begin{aligned}
\mu \int_{\tau_{i}} \frac{\mathbf{H}_{i}^{n+\frac{3}{2}}-\mathbf{H}_{i}^{n+\frac{1}{2}}}{\Delta t} \cdot \varphi_{h} & =\sum_{k \in \mathcal{V}_{i}} \int_{a_{i k}} \varphi_{h} \cdot\left(\left\{\mathbf{E}_{h}^{n+1}\right\}_{i k} \times \mathbf{n}_{i k}\right)-\int_{\tau_{i}} \operatorname{curl} \varphi_{h} \cdot \mathbf{E}_{i}^{n+1} \\
\varepsilon_{0} \varepsilon_{\infty} \int_{\tau_{i}} \frac{\mathbf{E}_{i}^{n+1}-\mathbf{E}_{i}^{n}}{\Delta t} \cdot \psi_{h} & =-\sum_{k \in \mathcal{V}_{i}} \int_{a_{i k}} \psi_{h} \cdot\left(\left\{\mathbf{H}_{h}^{n+\frac{1}{2}}\right\}_{i k} \times \mathbf{n}_{i k}\right)+\int_{\tau_{i}} \mathbf{c u r l} \psi_{h} \cdot \mathbf{H}_{i}^{n+\frac{1}{2}} \\
& +\frac{1}{\tau_{r}} \int_{\tau_{i}} \mathbf{P}_{i}^{\left[n+\frac{1}{2}\right]} \cdot \psi_{h}-\alpha \int_{\tau_{i}} \mathbf{E}_{i}^{\left[n+\frac{1}{2}\right]} \cdot \psi_{h} \\
\frac{1}{\varepsilon_{0}\left(\varepsilon_{s}-\varepsilon_{\infty}\right)} \int_{\tau_{i}} \frac{\mathbf{P}_{i}^{n+1}-\mathbf{P}_{i}^{n}}{\Delta t} \cdot \phi_{h} & =-\frac{1}{\varepsilon_{0}\left(\varepsilon_{s}-\varepsilon_{\infty}\right) \tau_{r}} \int_{\tau_{i}} \mathbf{P}_{i}^{\left[n+\frac{1}{2}\right]} \cdot \phi_{h}+\frac{1}{\tau_{r}} \int_{\tau_{i}} \mathbf{E}_{i}^{\left[n+\frac{1}{2}\right]} \cdot \phi_{h} .
\end{aligned}
$$

Initial conditions are given by $\mathbf{H}_{h}^{0}=\mathbf{H}_{h}(0), \mathbf{E}_{h}^{0}=\mathbf{E}_{h}(0), \mathbf{P}_{h}^{0}=\mathbf{P}_{h}(0)$.

The fact that the DG mass matrix is invertible uniquely defines, under the conditions:

$$
1+\frac{\Delta t}{2 \tau_{r}} \neq 0 \text { and } \varepsilon_{0} \varepsilon_{\infty}+\frac{\alpha}{2} \Delta t-\frac{\beta}{2 \tau_{r}+\Delta t} \neq 0
$$

the iterates $\left(\mathbf{H}^{n+\frac{1}{2}}, \mathbf{E}^{n+1}, \mathbf{P}^{n+1}\right)$.

Like in the semi-discrete case, we now turn to stability analysis via the study of the associated discrete energy.

\subsection{Discrete energy}

One defines:

$$
\mathcal{E}^{n}=\sum_{i \in\left[0, N_{\tau}\right]} \mathcal{E}_{i}^{n},
$$

with:

$$
\mathcal{E}_{i}^{n}=\frac{1}{2}\left(\varepsilon_{0} \varepsilon_{\infty}\left\langle\mathbf{E}_{i}^{n}, \mathbf{E}_{i}^{n}\right\rangle_{/ \tau_{i}}+\mu\left\langle\mathbf{H}_{i}^{n-\frac{1}{2}}, \mathbf{H}_{i}^{n+\frac{1}{2}}\right\rangle_{/ \tau_{i}}+\frac{1}{\varepsilon_{0}\left(\varepsilon_{s}-\varepsilon_{\infty}\right)}\left\langle\mathbf{P}_{i}^{n}, \mathbf{P}_{i}^{n}\right\rangle_{/ \tau_{i}}\right)
$$

where $\langle.,.\rangle / \tau_{i}$ denotes the scalar product on $\tau_{i}$.

Positivity of the energy. With the hypotheses made on the mesh elements $\tau_{i}$, we can assume that there exists a generic constant $C>0$ such that $\forall p \in \mathbb{P}_{k}$ :

$$
\|\operatorname{curl} p\|_{\tau_{i}} \leq C h^{-1}\|p\|_{\tau_{i}}
$$

and:

$$
\|p\|_{a_{i k}}^{2} \leq C h^{-1}\|p\|_{\tau_{i}}^{2} .
$$

One can then follow exactly [FLLP05] ( $\S 2.4$, the added term in the energy we consider does not affect the proof) to prove the following result:

Proposition 4.1 (CFL condition) The discrete energy 24)-25) defines a quadratic form of the unknowns $\mathbf{E}^{n}, \mathbf{H}^{n-\frac{1}{2}}$ and $\mathbf{P}^{n}$ if:

$$
\frac{1}{\sqrt{\varepsilon_{0} \varepsilon_{\infty} \mu}} \Delta t<C h .
$$


Variation of the energy. We now prove the following result.

Proposition 4.2 The discrete energy (24)-(25) is decreasing i.e. there exists $C>0$ such that:

$$
\mathcal{E}^{n} \leq \mathcal{E}^{0}
$$

Proof. One writes the difference $\mathcal{E}_{i}^{n+1}-\mathcal{E}_{i}^{n}$ and uses the fully discrete version of the equations with particular test functions. Equation (21) is used at time stations $n+\frac{1}{2}$ and at time $n-\frac{1}{2}$ with test function given by $\mathbf{H}_{i}^{n+\frac{1}{2}}$. Equation (22) is used at time station $n$ with test function given by $\mathbf{E}_{i}^{\left[n+\frac{1}{2}\right]}$. Equation (23) is used at time station $n$, with test function given by $\mathbf{P}_{i}^{\left[n+\frac{1}{2}\right]}$. This yields:

$$
\begin{aligned}
\mathcal{E}_{i}^{n+1}-\mathcal{E}_{i}^{n} & =-\Delta t \sum_{k \in \mathcal{V}_{i}} \int_{a_{i k}} \mathbf{E}_{i}^{\left[n+\frac{1}{2}\right]} \cdot\left(\left\{\mathbf{H}_{h}^{n+\frac{1}{2}}\right\}_{a_{i k}} \times \mathbf{n}_{i k}\right)+\Delta t \int_{\tau_{i}} \operatorname{curl}_{\mathbf{E}_{i}^{\left[n+\frac{1}{2}\right]} \cdot \mathbf{H}_{i}^{n+\frac{1}{2}}} \\
& +\frac{\Delta t}{\tau_{r}} \int_{\tau_{i}} \mathbf{P}_{i}^{\left[n+\frac{1}{2}\right]} \cdot \mathbf{E}_{i}^{\left[n+\frac{1}{2}\right]}-\alpha \Delta t \int_{\tau_{i}}\left|\mathbf{E}_{i}^{\left[n+\frac{1}{2}\right]}\right|^{2} \\
& +\Delta t \sum_{k \in \mathcal{V}_{i}} \int_{a_{i k}} \mathbf{H}_{i}^{n+\frac{1}{2}} \cdot\left(\left\{\mathbf{E}_{h}^{\left[n+\frac{1}{2}\right]}\right\}_{a_{i k}} \times \mathbf{n}_{i k}\right)-\Delta t \int_{\tau_{i}} \operatorname{curl} \mathbf{H}_{i}^{n+\frac{1}{2}} \cdot \mathbf{E}_{i}^{\left[n+\frac{1}{2}\right]} \\
& -\frac{\Delta t}{\varepsilon_{0}\left(\varepsilon_{s}-\varepsilon_{\infty}\right) \tau_{r}} \int_{\tau_{i}}\left|\mathbf{P}_{i}^{\left[n+\frac{1}{2}\right]}\right|^{2}+\frac{\Delta t}{\tau_{r}} \int_{\tau_{i}} \mathbf{E}_{i}^{\left[n+\frac{1}{2}\right]} \cdot \mathbf{P}_{i}^{\left[n+\frac{1}{2}\right]} .
\end{aligned}
$$

Integrating by parts yields:

$$
\begin{aligned}
\mathcal{E}_{i}^{n+1}-\mathcal{E}_{i}^{n} & =-\frac{\Delta t}{2} \sum_{k \in \mathcal{V}_{i}} \int_{a_{i k}}\left(\mathbf{E}_{i}^{\left[n+\frac{1}{2}\right]} \times \mathbf{H}_{k}^{n+\frac{1}{2}}+\mathbf{E}_{k}^{\left[n+\frac{1}{2}\right]} \times \mathbf{H}_{i}^{n+\frac{1}{2}}\right) \cdot \mathbf{n}_{i k} \\
& +\frac{\Delta t}{\tau_{r}} \int_{\tau_{i}} \mathbf{P}_{i}^{\left[n+\frac{1}{2}\right]} \cdot \mathbf{E}_{i}^{\left[n+\frac{1}{2}\right]}-\alpha \Delta t \int_{\tau_{i}}\left|\mathbf{E}_{i}^{\left[n+\frac{1}{2}\right]}\right|^{2} \\
& -\frac{\Delta t}{\varepsilon_{0}\left(\varepsilon_{s}-\varepsilon_{\infty}\right) \tau_{r}} \int_{\tau_{i}}\left|\mathbf{P}_{i}^{\left[n+\frac{1}{2}\right]}\right|^{2}+\frac{\Delta t}{\tau_{r}} \int_{\tau_{i}} \mathbf{E}_{i}^{\left[n+\frac{1}{2}\right]} \cdot \mathbf{P}_{i}^{\left[n+\frac{1}{2}\right]} .
\end{aligned}
$$

Taking into account that we are dealing with a metallic boundary condition only, and adding all the contributions:

$$
\begin{aligned}
\mathcal{E}^{n+1}-\mathcal{E}^{n=}=\sum_{i \in\left[0, N_{T}\right]}\left[\frac{2 \Delta t}{\tau_{r}} \int_{\tau_{i}} \mathbf{P}_{i}^{\left[n+\frac{1}{2}\right]} \cdot \mathbf{E}_{i}^{\left[n+\frac{1}{2}\right]}-\alpha \Delta t \int_{\tau_{i}}\left|\mathbf{E}_{i}^{\left[n+\frac{1}{2}\right]}\right|^{2}-\right. \\
\left.\frac{\Delta t}{\varepsilon_{0}\left(\varepsilon_{s}-\varepsilon_{\infty}\right) \tau_{r}} \int_{\tau_{i}}\left|\mathbf{P}_{i}^{\left[n+\frac{1}{2}\right]}\right|^{2}+\right] .
\end{aligned}
$$

This gives like in the continuous and semi-discrete case:

$$
\mathcal{E}^{n+1}-\mathcal{E}^{n} \leq 0
$$

We can now turn to the analysis of the convergence of the fully discrete scheme.

\subsection{Convergence of the fully discrete scheme}

In this section we prove the following result. 
Proposition 4.3 Let $(\mathbf{H}, \mathbf{E}, \mathbf{P}) \in \mathcal{C}^{3}([0, T], \mathcal{L}(\Omega)) \cap \mathcal{C}^{0}\left([0, T],\left(\mathbf{H}^{s+1}(\Omega)\right)^{3}\right)$. Under the CFL condition (26), the following error estimate holds:

$$
\begin{gathered}
\max _{n=0 . . N}\left(\mu\left\|\mathbf{H}\left(t_{n+\frac{1}{2}}\right)-\mathbf{H}_{h}^{n+\frac{1}{2}}\right\|_{\mathcal{C}\left([0, T], \mathbf{L}^{2}(\Omega)\right)}^{2}+\varepsilon_{0} \varepsilon_{\infty}\left\|\mathbf{E}\left(t_{n}\right)-\mathbf{E}_{h}^{n}\right\|_{\mathcal{C}\left([0, T], \mathbf{L}^{2}(\Omega)\right)}^{2}+\right. \\
\left.\frac{1}{\varepsilon_{0}\left(\varepsilon_{s}-\varepsilon_{\infty}\right)}\left\|\mathbf{P}\left(t_{n}\right)-\mathbf{P}_{h}^{n}\right\|_{\mathcal{C}\left([0, T], \mathbf{L}^{2}(\Omega)\right)}^{2}\right)^{\frac{1}{2}} \\
\leq C\left(\Delta t^{2}+h^{\min (s, k)}\right)\left(\|(\mathbf{H}, \mathbf{E}, \mathbf{P})\|_{\mathcal{C}^{3}([0, T], \mathcal{L}(\Omega))}+\|(\mathbf{H}, \mathbf{E}, \mathbf{P})\|_{\mathcal{C}^{0}\left([0, T],\left(\mathbf{H}^{s+1}(\Omega)\right)^{3}\right)}\right)
\end{gathered}
$$

Proof. The proof uses a result of consistency. It involves the estimation of the local consistency error given by:

$$
\varepsilon_{h}^{n}=\left(\left\|\mathbf{E}_{h}\left(t_{n+1}\right)-\tilde{\mathbf{E}}_{h}^{n+1}\right\|_{\mathbf{L}^{2}(\Omega)}^{2}+\left\|\mathbf{H}_{h}\left(t_{n+\frac{3}{2}}\right)-\tilde{\mathbf{H}}_{h}^{n+\frac{3}{2}}\right\|_{\mathbf{L}^{2}(\Omega)}^{2}+\left\|\mathbf{P}_{h}\left(t_{n+1}\right)-\tilde{\mathbf{P}}_{h}^{n+1}\right\|_{\mathbf{L}^{2}(\Omega)}^{2}\right)^{\frac{1}{2}}
$$

where $\tilde{\mathbf{E}}_{h}^{n+1}, \tilde{\mathbf{H}}_{h}^{n+\frac{3}{2}}, \tilde{\mathbf{P}}_{h}^{n+1}$ have been computed from equations (21), (22), (23) as follows:

$$
\begin{aligned}
\mu \int_{\tau_{i}} \frac{\tilde{\mathbf{H}}_{i}^{n+\frac{3}{2}}-\mathbf{H}_{i}\left(t_{n+\frac{1}{2}}\right)}{\Delta t} \cdot \varphi_{h} & =\sum_{k \in \mathcal{V}_{i}} \int_{a_{i k}} \varphi_{h} \cdot\left\{\mathbf{E}_{h}\left(t_{n+1}\right)\right\}_{i k} \times \mathbf{n}_{i k} \\
& -\int_{\tau_{i}} \operatorname{curl} \varphi_{h} \cdot \mathbf{E}_{i}\left(t_{n+1}\right) \\
\varepsilon_{0} \varepsilon_{\infty} \int_{\tau_{i}} \frac{\tilde{\mathbf{E}}_{i}^{n+1}-\mathbf{E}_{i}\left(t_{n}\right)}{\Delta t} \cdot \psi_{h} & =\sum_{k \in \mathcal{V}_{i}} \int_{a_{i k}} \psi_{h} \cdot\left\{\mathbf{H}_{h}\left(t_{n+\frac{1}{2}}\right)\right\}_{i k} \times \mathbf{n}_{i k} \\
& +\int_{\tau_{i}} \mathbf{c u r l} \psi_{h} \cdot \mathbf{H}_{i}\left(t_{n+\frac{1}{2}}\right)+\int_{\tau_{i}} \frac{\mathbf{P}_{i}\left(t_{n+1}\right)+\mathbf{P}_{i}\left(t_{n}\right)}{2 \tau_{r}} \cdot \psi_{h} \\
& -\alpha \int_{\tau_{i}} \frac{\mathbf{E}_{i}\left(t_{n+1}\right)+\mathbf{E}_{i}\left(t_{n}\right)}{2} \cdot \psi_{h}, \\
\int_{\tau_{i}} \frac{\tilde{\mathbf{P}}_{i}^{n+1}-\mathbf{P}_{i}\left(t_{n}\right)}{\Delta t} \cdot \phi_{h} & =-\frac{1}{\tau_{r}} \int_{\tau_{i}} \frac{\mathbf{P}_{i}\left(t_{n+1}\right)+\mathbf{P}_{i}\left(t_{n}\right)}{2} \cdot \phi_{h} \\
& +\beta \int_{\tau_{i}} \frac{\mathbf{E}_{i}\left(t_{n+1}\right)+\mathbf{E}_{i}\left(t_{n}\right)}{2} \cdot \phi_{h},
\end{aligned}
$$

For the theory on ordinary differential equations, we follow HLW06, Dem99. Using Taylor formulas and approximation properties, one can prove that the consistency error is of order 2 , by which we mean that there exists $C>0$, such that:

$$
\left|\varepsilon_{h}^{n}\right| \leq C \Delta t^{3}\|\mathbf{U}\|_{\mathcal{C}^{3}\left([0, T], \mathbf{L}^{2}(\Omega)\right)},
$$

Indeed, one uses equalities like:

$$
\mathbf{E}_{h}\left(t_{n+1}\right)-\mathbf{E}_{h}\left(t_{n}\right)=\Delta t \frac{\partial}{\partial t} \mathbf{E}_{h}\left(t_{n+\frac{1}{2}}\right)+\frac{\Delta t^{3}}{28}\left(\frac{\partial^{3}}{\partial t^{3}} \mathbf{E}_{h}\left(c_{n+1}\right)+\frac{\partial^{3}}{\partial t^{3}} \mathbf{E}_{h}\left(c_{n}\right)\right)
$$

and:

$$
\frac{\mathbf{E}_{h}\left(t_{n+1}\right)+\mathbf{E}_{h}\left(t_{n}\right)}{2}=\mathbf{E}\left(t_{n+\frac{1}{2}}\right)+\frac{\Delta t^{2}}{16}\left(\frac{\partial^{2}}{\partial t^{2}} \mathbf{E}_{h}\left(d_{n+1}\right)+\frac{\partial^{2}}{\partial t^{2}} \mathbf{E}_{h}\left(d_{n}\right)\right) .
$$

$\left(c_{n+1}, c_{n}\right) \in\left[t_{n+\frac{1}{2}}, t_{n+1}\right] \times\left[t_{n}, t_{n+\frac{1}{2}}\right]$ and $\left(d_{n+1}, d_{n}\right) \in\left[t_{n+\frac{1}{2}}, t_{n+1}\right] \times\left[t_{n}, t_{n+\frac{1}{2}}\right]$.

RR $n^{\circ} 7634$ 
Writing the local error, one obtains $\int_{\Omega}\left(\mathbf{E}_{h}\left(t_{n+1}\right)-\tilde{\mathbf{E}}_{h}^{n+1}\right) \cdot \psi_{h}$ in terms of integrals of the type $\Delta t^{3} \int_{\Omega} \frac{\partial^{3}}{\partial t^{3}} \mathbf{E}_{h} \cdot \psi_{h}, \Delta t^{3} \int_{\Omega} \frac{\partial^{2}}{\partial t^{2}} \mathbf{E}_{h} \cdot \psi_{h}, \Delta t^{3} \int_{\Omega} \frac{\partial^{2}}{\partial t^{2}} \mathbf{P}_{h} \cdot \psi_{h}$. This leads to the estimation:

$$
\left\|\mathbf{E}_{h}\left(t_{n+1}\right)-\tilde{\mathbf{E}}_{h}^{n+1}\right\|_{\mathbf{L}^{2}(\Omega)} \leq C \Delta t^{3}\left(\left\|\frac{\partial^{3}}{\partial t^{3}} \mathbf{E}_{h}\right\|_{\mathbf{L}^{2}(\Omega)}+\left\|\frac{\partial^{2}}{\partial t^{2}} \mathbf{E}_{h}\right\|_{\mathbf{L}^{2}(\Omega)}+\left\|\frac{\partial^{2}}{\partial t^{2}} \mathbf{P}_{h}\right\|_{\mathbf{L}^{2}(\Omega)}\right) .
$$

With same arguments for the remaining two equations, one obtains (35).

With this notation $\left(\mathbf{H}_{h}, \mathbf{E}_{h}, \mathbf{P}_{h}\right)$ verifies the following set of equations:

$$
\begin{aligned}
\mu \int_{\tau_{i}} \frac{\mathbf{H}_{i}\left(t_{n+\frac{3}{2}}\right)-\mathbf{H}_{i}\left(t_{n+\frac{1}{2}}\right)}{\Delta t} \cdot \varphi_{h} & =\sum_{k \in \mathcal{V}_{i}} \int_{a_{i k}} \varphi_{h} \cdot\left\{\mathbf{E}_{h}\left(t_{n+1}\right)\right\}_{i k} \times \mathbf{n}_{i k}-\int_{\tau_{i}} \operatorname{curl} \varphi_{h} \cdot \mathbf{E}_{i}\left(t_{n+1}\right) \\
& +\int_{\tau_{i}} \frac{\mu}{\Delta t}\left(\mathbf{H}_{i}\left(t_{n+\frac{3}{2}}\right)-\tilde{\mathbf{H}}_{i}^{n+\frac{3}{2}}\right) \cdot \varphi_{h}, \\
\varepsilon \int_{\tau_{i}} \frac{\mathbf{E}_{i}\left(t_{n+1}\right)-\mathbf{E}_{i}\left(t_{n}\right)}{\Delta t} \cdot \psi_{h} & =\sum_{k \in \mathcal{V}_{i}} \int_{a_{i k}} \psi_{h} \cdot\left\{\mathbf{H}_{h}\left(t_{n+\frac{1}{2}}\right)\right\}_{i k} \times \mathbf{n}_{i k}+\int_{\tau_{i}} \mathbf{c u r l} \psi_{h} \cdot \mathbf{H}_{i}\left(t_{n+\frac{1}{2}}\right) \\
& +\int_{\tau_{i}} \frac{\mathbf{P}_{i}\left(t_{n}\right)+\mathbf{P}_{i}\left(t_{n+1}\right)}{2 \tau_{r}} \cdot \psi_{h}-\alpha \int_{\tau_{i}} \frac{\mathbf{E}_{i}\left(t_{n}\right)+\mathbf{E}_{i}\left(t_{n+1}\right)}{2} \cdot \psi_{h} \\
& +\int_{\tau_{i}} \frac{\varepsilon_{0} \varepsilon_{\infty}}{\Delta t}\left(\mathbf{E}_{i}\left(t_{n+1}\right)-\tilde{\mathbf{E}}_{i}^{n+1}\right) \cdot \psi_{h}, \\
& =-\frac{1}{\tau_{r}} \int_{\tau_{i}} \frac{\mathbf{P}_{i}\left(t_{n}\right)+\mathbf{P}_{i}\left(t_{n+1}\right)}{2} \cdot \phi_{h}+\beta \int_{\tau_{i}} \frac{\mathbf{E}_{i}\left(t_{n}\right)+\mathbf{E}_{i}\left(t_{n+1}\right)}{2} \cdot \phi_{h} \\
\int_{\tau_{i}} \frac{\mathbf{P}_{i}\left(t_{n+1}\right)-\mathbf{P}_{i}\left(t_{n}\right)}{\Delta t} \cdot \phi_{h} & +\int_{\tau_{i}} \frac{1}{\Delta t}\left(\mathbf{P}_{i}\left(t_{n+1}\right)-\tilde{\mathbf{P}}_{i}^{n+1}\right) \cdot \phi_{h},
\end{aligned}
$$

So that if one defines:

$$
\begin{aligned}
\mathfrak{h}_{i}^{n}\left(\varphi_{h}\right) & =\int_{\tau_{i}} \frac{\mu}{\Delta t}\left(\mathbf{H}_{i}\left(t_{n+\frac{3}{2}}\right)-\tilde{\mathbf{H}}_{i}^{n+\frac{3}{2}}\right) \cdot \varphi_{h}, \\
\mathfrak{e}_{i}^{n}\left(\psi_{h}\right) & =\int_{\tau_{i}} \frac{\varepsilon_{0} \varepsilon_{\infty}}{\Delta t}\left(\mathbf{E}_{i}\left(t_{n+1}\right)-\tilde{\mathbf{E}}_{i}^{n+1}\right) \cdot \psi_{h}, \\
\mathfrak{p}_{i}^{n}\left(\phi_{h}\right) & =\int_{\tau_{i}} \frac{1}{\Delta t}\left(\mathbf{P}_{i}\left(t_{n+1}\right)-\tilde{\mathbf{P}}_{i}^{n+1}\right) \cdot \phi_{h},
\end{aligned}
$$

one has:

$$
\begin{aligned}
\mu \int_{\tau_{i}} \frac{\mathbf{H}_{i}\left(t_{n+\frac{3}{2}}\right)-\mathbf{H}_{i}\left(t_{n+\frac{1}{2}}\right)}{\Delta t} \cdot \varphi_{h} & =\sum_{k \in \mathcal{V}_{i}} \int_{a_{i k}} \varphi_{h} \cdot\left\{\mathbf{E}_{h}\left(t_{n+1}\right)\right\}_{i k} \times \mathbf{n}_{i k} \\
& -\int_{\tau_{i}} \operatorname{curl} \varphi_{h} \cdot \mathbf{E}_{i}\left(t_{n+1}\right)+\mathfrak{h}_{i}^{n}\left(\varphi_{h}\right), \\
\varepsilon_{0} \varepsilon_{\infty} \int_{\tau_{i}} \frac{\mathbf{E}_{i}\left(t_{n+1}\right)-\mathbf{E}_{i}\left(t_{n}\right)}{\Delta t} \cdot \psi_{h} & =\sum_{k \in \mathcal{V}_{i}} \int_{a_{i k}} \psi_{h} \cdot\left\{\mathbf{H}_{h}\left(t_{n+\frac{1}{2}}\right)\right\}_{i k} \times \mathbf{n}_{i k} \\
& +\int_{\tau_{i}} \operatorname{curl} \psi_{h} \cdot \mathbf{H}_{i}\left(t_{n+\frac{1}{2}}\right)+\int_{\tau_{i}} \frac{\mathbf{P}_{i}\left(t_{n}\right)+\mathbf{P}_{i}\left(t_{n+1}\right)}{2 \tau_{r}} \cdot \psi_{h} \\
& -\alpha \int_{\tau_{i}} \frac{\mathbf{E}_{i}\left(t_{n}\right)+\mathbf{E}_{i}\left(t_{n+1}\right)}{2} \cdot \psi_{h}+\mathfrak{e}_{i}^{n}\left(\psi_{h}\right), \\
& =-\frac{1}{\tau_{r}} \int_{\tau_{i}} \frac{\mathbf{P}_{i}\left(t_{n}\right)+\mathbf{P}_{i}\left(t_{n+1}\right)}{2} \cdot \phi_{h} \\
\int_{\tau_{i}} \frac{\mathbf{P}_{i}\left(t_{n+1}\right)-\mathbf{P}_{i}\left(t_{n}\right)}{\Delta t} \cdot \phi_{h} & +\beta \int_{\tau_{i}} \frac{\mathbf{E}_{i}\left(t_{n}\right)+\mathbf{E}_{i}\left(t_{n+1}\right)}{2} \cdot \phi_{h}+\mathfrak{p}_{i}^{n}\left(\phi_{h}\right),
\end{aligned}
$$


In a global formulation:

$$
\begin{aligned}
\mathfrak{h}_{h}^{n}\left(\varphi_{h}\right) & =\int_{\Omega} \frac{\mu}{\Delta t}\left(\mathbf{H}_{h}\left(t_{n+\frac{3}{2}}\right)-\tilde{\mathbf{H}}_{h}^{n+\frac{3}{2}}\right) \cdot \varphi_{h}, \\
\mathfrak{e}_{h}^{n}\left(\psi_{h}\right) & =\int_{\Omega} \frac{\varepsilon_{0} \varepsilon_{\infty}}{\Delta t}\left(\mathbf{E}_{h}\left(t_{n+1}\right)-\tilde{\mathbf{E}}_{h}^{n+1}\right) \cdot \psi_{h}, \\
\mathfrak{p}_{h}^{n}\left(\phi_{h}\right) & =\int_{\Omega} \frac{1}{\Delta t}\left(\mathbf{P}_{h}\left(t_{n+1}\right)-\tilde{\mathbf{P}}_{h}^{n+1}\right) \cdot \phi_{h} .
\end{aligned}
$$

and

$$
\begin{aligned}
\left|\mathfrak{h}_{h}^{n}\left(\varphi_{h}\right)\right| & \leq \frac{C}{\Delta t}\left\|\mathbf{H}_{h}\left(t_{n+\frac{3}{2}}\right)-\tilde{\mathbf{H}}_{h}^{n+\frac{3}{2}}\right\|_{\mathbf{L}^{2}(\Omega)}\left\|\varphi_{h}\right\|_{\mathbf{L}^{2}(\Omega)}, \\
\left|\mathfrak{e}_{h}^{n}\left(\psi_{h}\right)\right| & \leq \frac{C}{\Delta t}\left\|\mathbf{E}_{h}\left(t_{n+1}\right)-\tilde{\mathbf{E}}_{h}^{n+1}\right\|_{\mathbf{L}^{2}(\Omega)}\left\|\psi_{h}\right\|_{\mathbf{L}^{2}(\Omega)}, \\
\left|\mathfrak{p}_{h}^{n}\left(\phi_{h}\right)\right| & \leq \frac{C}{\Delta t}\left\|\mathbf{P}_{h}\left(t_{n+1}\right)-\tilde{\mathbf{P}}_{h}^{n+1}\right\|_{\mathbf{L}^{2}(\Omega)}\left\|\phi_{h}\right\|_{\mathbf{L}^{2}(\Omega)} .
\end{aligned}
$$

Using (35), one has:

$$
\left\||| \mathfrak{h}_{h}^{n}|||+||| \mathfrak{e}_{h}^{n}|||+||| \mathfrak{p}_{h}^{n}|| \mid \leq C \Delta t^{2}\right\| \mathbf{U} \|_{\mathcal{C}^{3}\left([0, T], \mathbf{L}^{2}(\Omega)\right)},
$$

where $\||\cdot|\|$ is these linear forms norm if one considers the linear form on $\mathbf{L}^{2}(\Omega)$. With this at hand, one can study the error. Let:

$$
\eta_{h}^{n}=\mathbf{U}_{h}\left(t_{n}\right)-\mathbf{U}_{h}^{n}=\left(\mathbb{H}_{h}^{n-\frac{1}{2}}, \mathbb{E}_{h}^{n}, \mathbb{P}_{h}^{n}\right)
$$

and:

$$
\begin{gathered}
\mathbb{H}_{h}^{n-\frac{1}{2}}=\mathbf{H}_{h}\left(t_{n-\frac{1}{2}}\right)-\mathbf{H}_{h}^{n-\frac{1}{2}}, \\
\mathbb{E}_{h}^{n}=\mathbf{E}_{h}\left(t_{n}\right)-\mathbf{E}_{h}^{n}, \quad \mathbb{P}_{h}^{n}=\mathbf{P}_{h}\left(t_{n}\right)-\mathbf{P}_{h}^{n}
\end{gathered}
$$

Define the error energy:

$$
\mathfrak{E}_{i}^{n}=\frac{1}{2}\left(\varepsilon_{0} \varepsilon_{\infty}\left\|\mathbb{E}_{i}^{n}\right\|_{\mathbf{L}^{2}\left(\tau_{i}\right)}^{2}+\mu\left\langle\mathbb{H}_{i}^{n+\frac{1}{2}}, \mathbb{H}_{i}^{n-\frac{1}{2}}\right\rangle_{/ \tau_{i}}+\frac{1}{\varepsilon_{0}\left(\varepsilon_{s}-\varepsilon_{\infty}\right)}\left\|\mathbb{P}_{i}^{n}\right\|_{\mathbf{L}^{2}\left(\tau_{i}\right)}^{2}\right) .
$$

The fields $\left(\mathbb{H}_{h}^{n}, \mathbb{E}_{h}^{n}, \mathbb{P}_{h}^{n}\right)$ verify the equations:

$$
\begin{aligned}
\mu \int_{\tau_{i}} \frac{\mathbb{H}_{i}^{n+\frac{3}{2}}-\mathbb{H}_{i}^{n+\frac{1}{2}}}{\Delta t} \cdot \varphi_{h} & =\sum_{k \in \mathcal{V}_{i}} \int_{a_{i k}} \varphi_{h} \cdot\left\{\mathbb{E}_{h}^{n+1}\right\}_{i k} \times \mathbf{n}_{i k} \\
& -\int_{\tau_{i}} \operatorname{curl} \varphi_{h} \cdot \mathbb{E}_{i}^{n+1}+\mathfrak{h}_{i}^{n}\left(\varphi_{h}\right) \\
\varepsilon \int_{\tau_{i}} \frac{\mathbb{E}_{i}^{n+1}-\mathbb{E}_{i}^{n}}{\Delta t} \cdot \psi_{h} & =\sum_{k \in \mathcal{V}_{i}} \int_{a_{i k}} \psi_{h} \cdot\left\{\mathbb{H}_{h}^{n+\frac{1}{2}}\right\}_{i k} \times \mathbf{n}_{i k} \\
& +\int_{\tau_{i}} \operatorname{curl} \psi_{h} \cdot \mathbb{H}_{i}^{n+\frac{1}{2}}+\int_{\tau_{i}} \frac{\mathbb{P}_{i}^{n}+\mathbb{P}_{i}^{n+1}}{2 \tau_{r}} \cdot \psi_{h} \\
& -\alpha \int_{\tau_{i}} \frac{\mathbb{E}_{i}^{n}+\mathbb{E}_{i}^{n+1}}{2} \cdot \psi_{h}+\mathfrak{e}_{i}^{n}\left(\psi_{h}\right), \\
\int_{\tau_{i}} \frac{\mathbb{P}_{i}^{n+1}-\mathbb{P}_{i}^{n} \cdot \phi_{h}}{\Delta t} & -\frac{1}{\tau_{r}} \int_{\tau_{i}} \frac{\mathbb{P}_{i}^{n}+\mathbb{P}_{i}^{n+1}}{2} \cdot \phi_{h} \\
& +\beta \int_{\tau_{i}} \frac{\mathbb{E}_{i}^{n}+\mathbb{E}_{i}^{n+1}}{2} \cdot \phi_{h}+\mathfrak{p}_{i}^{n}\left(\phi_{h}\right) .
\end{aligned}
$$

RR $n^{\circ} 7634$ 
Reasonning similarly than for the discrete energy:

$$
\begin{aligned}
& \mathfrak{E}^{n+1}-\mathfrak{E}^{n=} \sum_{i \in\left[0, N_{T}\right]}\left[\frac{\Delta t}{\tau_{r}} \int_{\tau_{i}} \mathbb{P}_{h}^{\left[n+\frac{1}{2}\right]} \cdot \mathbb{E}_{h}^{\left[n+\frac{1}{2}\right]}-\alpha \Delta t \int_{\tau_{i}}\left|\mathbb{E}_{h}^{\left[n+\frac{1}{2}\right]}\right|^{2}-\right. \\
&\left.\frac{\Delta t}{\varepsilon_{0}\left(\varepsilon_{s}-\varepsilon_{\infty}\right) \tau_{r}} \int_{\tau_{i}}\left|\mathbb{E}_{h}^{\left[n+\frac{1}{2}\right]}\right|^{2}+\frac{\Delta t}{\tau_{r}} \int_{\tau_{i}} \mathbb{E}_{h}^{\left[n+\frac{1}{2}\right]} \cdot \mathbb{P}_{h}^{\left[n+\frac{1}{2}\right]}\right] \\
&+\frac{1}{2}\left(\mathfrak{h}_{h}^{n}\left(\mathbb{H}^{n+\frac{1}{2}}\right)+\mathfrak{h}_{h}^{n+1}\left(\mathbb{H}^{n+\frac{1}{2}}\right)\right)+\mathfrak{e}_{h}^{n}\left(\mathbb{E}^{\left[n+\frac{1}{2}\right]}\right)+\mathfrak{p}_{h}^{n}\left(\mathbb{P}^{\left[n+\frac{1}{2}\right]}\right) .
\end{aligned}
$$

One can then prove that under a CFL condition of the same type than (26),

$$
\left(\left\|\mathbb{H}_{h}^{n}\right\|_{\mathbf{L}^{2}(\Omega)}^{2}+\left\|\mathbb{E}_{h}^{n}\right\|_{\mathbf{L}^{2}(\Omega)}^{2}+\left\|\mathbb{P}_{h}^{n}\right\|_{\mathbf{L}^{2}(\Omega)}^{2}\right)^{\frac{1}{2}} \leq \Delta t^{2}\|\mathbf{U}\|_{\mathcal{C}^{3}\left([0, T], \mathbf{L}^{2}(\Omega)\right)}
$$

This gives the result.

Using the triangle inequality and results already established in the semi-discrete case, one can deduce an error estimate:

$$
\begin{gathered}
\max _{n=0 . . N}\left(\mu\left\|\mathbf{H}\left(t_{n+\frac{1}{2}}\right)-\mathbf{H}_{h}^{n+\frac{1}{2}}\right\|_{\mathcal{C}\left([0, T], \mathbf{L}^{2}(\Omega)\right)}^{2}+\varepsilon_{0} \varepsilon_{\infty}\left\|\mathbf{E}\left(t_{n}\right)-\mathbf{E}_{h}^{n}\right\|_{\mathcal{C}\left([0, T], \mathbf{L}^{2}(\Omega)\right)}^{2}+\right. \\
\left.\frac{1}{\varepsilon_{0}\left(\varepsilon_{s}-\varepsilon_{\infty}\right)}\left\|\mathbf{P}\left(t_{n}\right)-\mathbf{P}_{h}^{n}\right\|_{\mathcal{C}\left([0, T], \mathbf{L}^{2}(\Omega)\right)}^{2}\right)^{\frac{1}{2}} \\
\leq C \Delta t^{2}\|(\mathbf{H}, \mathbf{E}, \mathbf{P})\|_{\mathcal{C}^{3}\left([0, T], \mathbf{L}^{2}(\Omega)\right)}+C h^{\min (s, k)}\|(\mathbf{H}, \mathbf{E}, \mathbf{P})\|_{\mathcal{C}\left([0, T],\left(\mathbf{H}^{s+1}(\Omega)\right)\right)^{3}} \\
\leq C\left(\Delta t^{2}+h^{\min (s, k)}\right)\left(\|(\mathbf{H}, \mathbf{E}, \mathbf{P})\|_{\mathcal{C}^{3}\left([0, T], \mathbf{L}^{2}(\Omega)\right)}+\|(\mathbf{H}, \mathbf{E}, \mathbf{P})\|_{\mathcal{C}^{0}\left([0, T],\left(\mathbf{H}^{s+1}(\Omega)\right)^{3}\right)}\right) .
\end{gathered}
$$

Finally, one can conclude that the order of the convergence of the scheme is $\mathcal{O}\left(\Delta t^{2}+h^{\min (s, k)}\right)$.

\section{$5 \quad$ Numerical results}

We present here some numerical results validating the theoretical analysis of the previous sections. We consider solving the following system of equations where one adds an artificial source current $\mathbf{J}$ in the equation of $\mathbf{E}$ which is such that we can deduce the exact solution of the problem:

$$
\left\{\begin{array}{l}
\mu \frac{\partial \mathbf{H}}{\partial t}+\operatorname{curl} \mathbf{E}=0 \\
\varepsilon_{0} \varepsilon_{\infty} \frac{\partial \mathbf{E}}{\partial t}-\operatorname{curl} \mathbf{H}=-\frac{1}{\tau_{r}}\left[\varepsilon_{0}\left(\varepsilon_{s}-\varepsilon_{\infty}\right) \mathbf{E}-\mathbf{P}\right]-\sigma \mathbf{E}+\mathbf{J} \\
\frac{\partial \mathbf{P}}{\partial t}=\frac{1}{\tau_{r}}\left[\varepsilon_{0}\left(\varepsilon_{s}-\varepsilon_{\infty}\right) \mathbf{E}-\mathbf{P}\right] .
\end{array}\right.
$$

The type of analysis used in previous sections extend to this case. Indeed, one can for example prove that:

which gives:

$$
\frac{d}{d t} \mathcal{E}(t) \leq C\|\mathbf{J}\|_{\mathbf{L}^{2}(\Omega)} \sqrt{\mathcal{E}(t)}
$$

$$
\sqrt{\mathcal{E}(t)} \leq \sqrt{\mathcal{E}(0)}+C T\|\mathbf{J}\|_{\mathcal{C}\left([0, T], \mathbf{L}^{2}(\Omega)\right)}
$$

so that the energy is bounded, but not decreasing. This type of argument is also used to prove an analoguous result on the boundedness on the semi-discrete energy. Furthermore, up to considering the 
projection of the source current on the finite element space, theorem 3.6 is still valid. We furthermore modify the fully-discrete scheme in accordance with the introduction of the source current. The modified scheme is as follows:

$$
\begin{aligned}
\mu_{\infty} \frac{\mathbf{H}_{i}^{n+\frac{3}{2}}-\mathbf{H}_{i}^{n+\frac{1}{2}}}{\Delta t} \cdot \varphi_{h} & =\sum_{k \in \mathcal{V}_{i}} \int_{a_{i k}} \varphi_{h} \cdot\left(\left\{\mathbf{E}_{h}^{n+1}\right\}_{i k} \times \mathbf{n}_{i k}\right)-\int_{\tau_{i}} \operatorname{curl} \varphi_{h} \cdot \mathbf{E}_{i}^{n+1}, \\
\varepsilon_{\infty} \int_{\tau_{i}} \frac{\mathbf{E}_{i}^{n+1}-\mathbf{E}_{i}^{n}}{\Delta t} \cdot \psi_{h} & =-\sum_{k \in \mathcal{V}_{i}} \int_{a_{i k}} \psi_{h} \cdot\left(\left\{\mathbf{H}_{h}^{n+\frac{1}{2}}\right\}_{i k} \times \mathbf{n}_{i k}\right)+\int_{\tau_{i}} \operatorname{curl} \psi_{h} \cdot \mathbf{H}_{i}^{n+\frac{1}{2}} \\
& +\frac{1}{\tau_{r}} \int_{\tau_{i}} \mathbf{P}_{i}^{\left[n+\frac{1}{2}\right]} \cdot \psi_{h}-\alpha \int_{\tau_{i}} \mathbf{E}_{i}^{\left[n+\frac{1}{2}\right]} \cdot \psi_{h}+\int_{\tau_{i}} \mathbf{J}_{i}^{n+\frac{1}{2}} \cdot \psi_{h}, \\
\int_{\tau_{i}} \frac{\mathbf{P}_{i}^{n+1}-\mathbf{P}_{i}^{n}}{\Delta t} \cdot \phi_{h} & =-\frac{1}{\tau_{r}} \int_{\tau_{i}} \mathbf{P}_{i}^{\left[n+\frac{1}{2}\right]} \cdot \phi_{h}+\beta \int_{\tau_{i}} \mathbf{E}_{i}^{\left[n+\frac{1}{2}\right]} \cdot \phi_{h} .
\end{aligned}
$$

with $\mathbf{J}_{i}^{n+\frac{1}{2}}=\mathbf{J}_{i}\left(t_{n+\frac{1}{2}}\right)$. The choice of considering $\mathbf{J}^{n+\frac{1}{2}}$ in the equations ensures that one keeps a scheme of second order accuracy. We choose to give the equation in an adimensional form with:

$$
\begin{gathered}
\tilde{\mathbf{H}}=\sqrt{\frac{\mu_{0}}{\varepsilon_{0}}} \mathbf{H}, \quad \tilde{\sigma}=\sqrt{\frac{\mu_{0}}{\varepsilon_{0}}} \sigma, \quad \tilde{\mathbf{E}}=\mathbf{E}, \quad \tilde{\mathbf{P}}=\frac{\mathbf{P}}{\varepsilon_{0}}, \\
\tilde{t}=\frac{1}{\sqrt{\varepsilon_{0} \mu_{0}}} t, \quad \tilde{\tau}_{r}=\frac{1}{\varepsilon_{0} \mu_{0}} \tau_{r}, \quad \tilde{\beta}=\frac{\varepsilon_{s}-\varepsilon_{\infty}}{\tilde{\tau}_{r}}, \quad \tilde{\alpha}=\tilde{\beta}+\tilde{\sigma}
\end{gathered}
$$

Furthermore, for this numerical test, we choose to work with Maxwell's equations in 2D for transverse magnetic (TMz) waves. In this case, one has that $H_{z}=E_{x}=E_{y}=P_{x}=P_{y}=0$ and the fields do not depend on the $z$ coordinate. The corresponding renormalized equations are then given by:

$$
\left\{\begin{array}{l}
\mu_{\infty} \frac{\partial \tilde{H}_{x}}{\partial \tilde{t}}+\frac{\partial \tilde{E}_{z}}{\partial y}=0 \\
\mu_{\infty} \frac{\partial \tilde{H}_{y}}{\partial \tilde{t}}-\frac{\partial \tilde{E}_{z}}{\partial x}=0 \\
\varepsilon_{\infty} \frac{\partial \tilde{E}_{z}}{\partial \tilde{t}}-\frac{\partial \tilde{H}_{y}}{\partial x}+\frac{\partial \tilde{H}_{x}}{\partial y}=\frac{1}{\tilde{\tau}_{r}} \tilde{P}_{z}-\tilde{\alpha} \tilde{E}_{z}+\tilde{J}_{z} \\
\frac{\partial \tilde{P}_{z}}{\partial \tilde{t}}=-\frac{1}{\tilde{\tau}_{r}} \tilde{P}_{z}+\tilde{\beta} \tilde{E}_{z}
\end{array}\right.
$$

where $\tilde{J}_{z}$ denotes the source current.

In the rest of this section, we will omit the . for clarity. The computational domain is the square $[0,1] \times[0,1]$. In our simulations, we choose $\mu_{\infty}=1, \varepsilon_{\infty}=1, \varepsilon_{s}=5, \tau_{r}=2.82 \times 10^{-3}$. The exact solution is given by:

$\mathrm{RR} \mathrm{n}^{\circ} 7634$

$$
\left\{\begin{array}{l}
H_{x}=-\frac{1}{\sqrt{2}} \sin (\pi x) \cos (\pi y) \sin (\sqrt{2} \pi t) \\
H_{y}=\frac{1}{\sqrt{2}} \cos (\pi x) \sin (\pi y) \sin (\sqrt{2} \pi t) \\
E_{z}=\sin (\pi x) \sin (\pi y) \cos (\sqrt{2} \pi t) \\
P_{z}=\beta \sin (\pi x) \sin (\pi y)\left[\frac{1}{4 \pi^{2}+\frac{1}{\tau_{r}^{2}}}\left(\frac{1}{\tau_{r}} \cos (\sqrt{2} \pi t)+\sqrt{2} \pi \sin (\sqrt{2} \pi t)\right)\right]
\end{array}\right.
$$


if we impose:

$$
\begin{aligned}
J_{z} & =-\frac{\beta}{\tau_{r}} \sin (\pi x) \sin (\pi y)\left[\frac{1}{4 \pi^{2}+\frac{1}{\tau_{r}^{2}}}\left(\frac{1}{\tau_{r}} \cos (\sqrt{2} \pi t)+\sqrt{2} \pi \sin (\sqrt{2} \pi t)\right)\right] \\
& +\alpha \sin (\pi x) \sin (\pi y) \cos (\sqrt{2} \pi t) .
\end{aligned}
$$

The above exact solution verifies the boundary conditions imposed to $\mathbf{H}$ and $\mathbf{E}$ on the border of the square. The simulation time has been fixed to $T=6 \mathrm{~m}$ (renormalized unit). The square is meshed with triangles by a subdivision of a finite different grid defined by the number of discretization points along the $x$ and $y$ axes. Our numerical tests are obtained using a Fortran 77 code. On figure 1 , we show the exact and computed solutions at the point with coordinates $(0.45,0.45)$ in the square. In table 1 and table 2 we present the energy errors obtained for the DGTD- $\mathbb{P}_{p}$ for $p=1$ and $p=2$ respectively. We choose the time step $\Delta t$ so that it verifies the CFL condition. In this manner, the convergence rate obtained corresponds to the space convergence rate. Indeed, since the theoretical convergence rate is $O\left(\Delta t^{2}+h^{p}\right)$, for a $\mathbb{P}_{p}$ interpolation with $p \leq 2$, the global convergence rate should be dominated by the one in space. This is what we observe in the present simulation results. Considering $p>3$ will lead to a rate of convergence of 2 since then the latter is dominated by the one in time. The numerical results are then in accordance with the prediction of the theoretical analysis.

\begin{tabular}{cccc}
\hline Mesh size & Energy error & CPU time (seconds) & Convergence rate \\
\hline $1 / 8$ & 0.062489 & 0.05 & - \\
$1 / 16$ & 0.028823 & 0.22 & 1.1164 \\
$1 / 32$ & 0.014248 & 1.63 & 1.0164 \\
$1 / 64$ & 0.007102 & 13.49 & 1.0045 \\
$1 / 128$ & 0.003546 & 128.77 & 1.0018 \\
\hline
\end{tabular}

Table 1: Energy errors obtained with $\Delta t=\mathrm{CFL} \times h$ for DGTD- $\mathbb{P}_{1}$ approximation.

\begin{tabular}{cccc}
\hline Mesh size & Energy error & CPU time (seconds) & Convergence rate \\
\hline $1 / 8$ & 0.00405208 & 0.08 & - \\
$1 / 16$ & 0.000898612 & 0.53 & 2.1729 \\
$1 / 32$ & 0.000220122 & 4.14 & 2.0294 \\
$1 / 64$ & $5.51758 \mathrm{e}-05$ & 35.87 & 1.9962 \\
$1 / 128$ & $1.38308 \mathrm{e}-05$ & 510.31 & 1.9961 \\
\hline
\end{tabular}

Table 2: Energy errors obtained with $\Delta t=\mathrm{CFL} \times h$ for DGTD- $\mathbb{P}_{2}$ approximation.

\section{Conclusion}

In this work we have conducted a complete study of a discontinuous Galerkin finite element formulation for Maxwell's equation in dispersive media. Some numerical results validate the theoretical study. Several such studies, either with continuous finite element methods or with discontinuous Galerkin methods, have been published in the recent years denoting an increased interest in this modeling context especially for the simulation of problems involving the interaction of electromagnetic waves with biological media. This work is a necessary first step in the study of Maxwell's equation in dispersive media, before exploiting this in future work, especially on more realistic 3D simulations. A typical application context that we will consider in the sequel is the numerical modelling of human head tissue exposure to electromagnetic waves from cellular phones $\left[\mathrm{SCL}^{+} 06\right]$. 

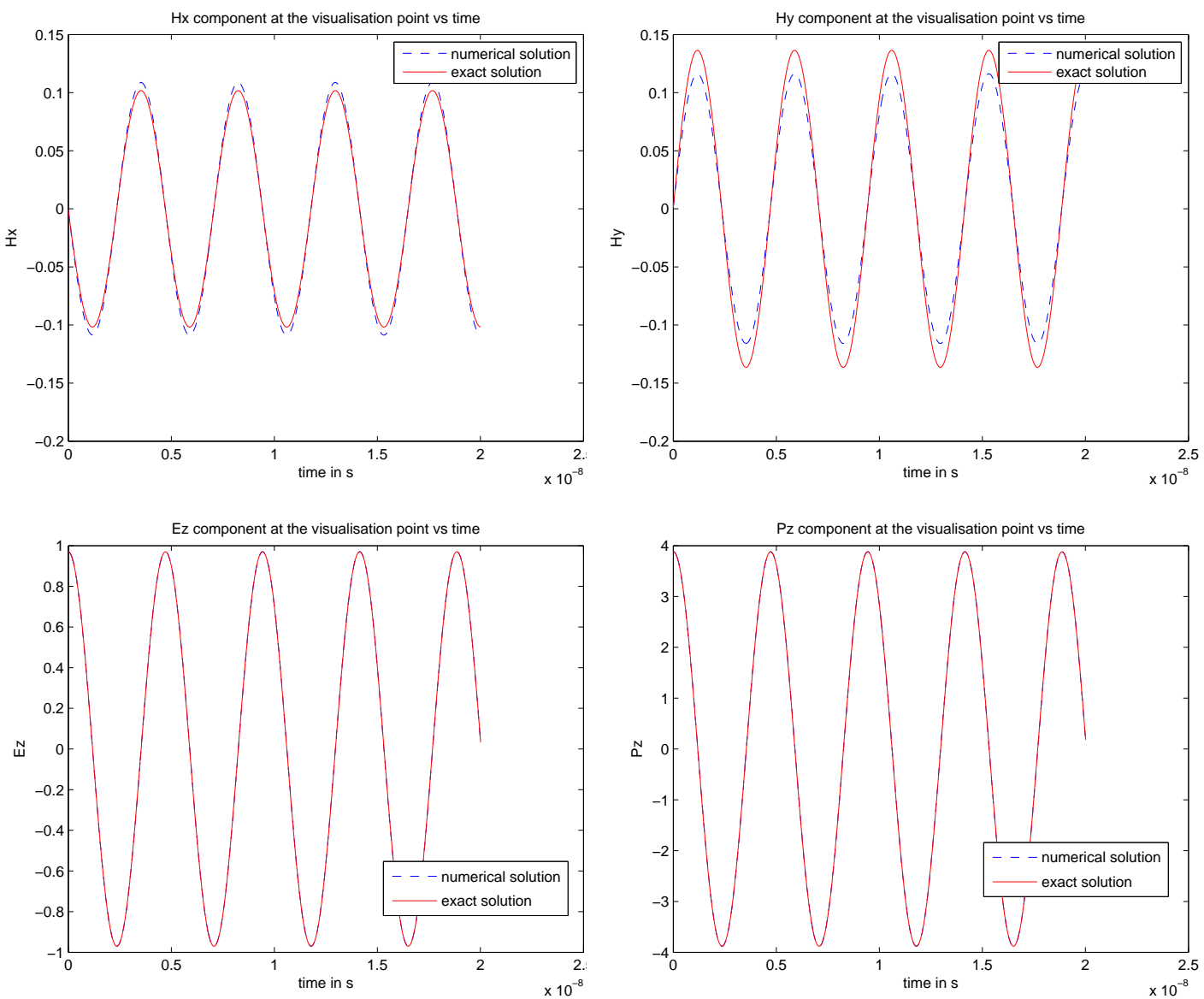

Figure 1: Comparison between exact and numerical solutions with $h=1 / 64$ and a DGTD- $\mathbb{P}_{2}$ approximation. 


\section{References}

[Bos98] A. Bossavit, Computational electromagnetism, Academic Press Series in Electromagnetism, Academic Press, 1998.

[CBHV04] M. Converse, E.J. Bond, S.C. Hagness, and B.D. Van Veen, Ultrawide-band microwave space-time beamforming for hyperthermia treatment of breast cancer: a computational feasability study, IEEE Trans. Microwave Theory Tech. 52 (2004), no. 8, 1876-1889.

[CH90] T. Cazenave and A. Haraux, Introduction aux problèmes d'évolution semi-linéaires, Mathematics and Applications, Ellipses, 1990.

[Dem99] J.P. Demailly, Analyse numérique et equations différentielle, Presses Universitaires de Grenoble, Grenoble Sciences, 1999.

[FLLP05] L. Fezoui, S. Lanteri, S. Lohrengel, and S. Piperno, Convergence and stability of a discontinuous Galerkin time-domain method for the 3D heterogeneous Maxwell equations on unstructuredmeshes, ESAIM: Math. Model. Numer. Anal. 39 (2005), no. 6, 1149-1176.

[HL09] Y. Huang and J. Li, Interior penalty discontinuous Galerkin method for Maxwell's equations in cold plasma, J. Sci. Comput. 41 (2009), no. 3, 321-340.

[HLW06] E. Hairer, C. Lubich, and G. Wanner, Geometric numerical integration. Structure preserving algorithms for ordinary differential equations, Computational Mathematics, vol. 31, Springer-Verlag, 2006.

[HLY11] Y. Huang, J. Li, and W. Yang, Interior penalty discontinuous Galerkin methods for Maxwell's equations in dispersive media, J. Comput. Phys. 230 (2011), no. 12, 4559-4570.

[KF90] T. Kashiwa and I. Fukai, A treatment by FD-TD method of the dispersive characteristics associated with electronic polarization, Tech. report, Departement of Electrical Engineering, Faculty of Engineering, Hokkaido University, 1990.

[KKGU08] I.S. Karanasiou, K.T. Karathanasis, A. Garetos, and N.K. Uzunoglu, Development and laboratory testing of a noninvasive intracranial focused hyperthermia system, IEEE Trans. Microwave Theory Tech. 56 (2008), no. 9, 2160-2171.

$\left[\mathrm{KLG}^{+} 10\right]$ M. Klemm, J.A. Leendertz, D.R. Gibbins, I.J. Craddock, A.W. Preece, and R. Benjamin, Towards contrast enhanced breast imaging using ultra-wideband microwave radar system, IEEE Radio and Wireless Symposium. Special session on biomedical applications (New Orleans, USA), 2010, pp. 516-519.

[LC06] J. Li and Y. Chen, Analysis of a time-domain finite element method for 3-D Maxwell's equations in dispersive media, Comput. Methods. Appl. Mech. Engrg. 195 (2006), no. 3336, 4220-4229.

[LC08] _ Finite element study of time-dependent Maxwell's equations in dispersive media, Numer. Meth. Part. Differ. Equ. 24 (2008), no. 5, 1203-1221.

[LCZ05] T. Lu, W. Cai, and P. Zhang, Discontinuous Galerkin time domain methods for GPR simulation in dispersive media, IEEE Trans. Geosc. Rem. Sens. 43 (2005), no. 1, 72-80.

[Li06] J. Li, Error analysis of finite element methods for 3-D Maxwell's equations in dispersive media, J. Comput. Appl. Math. 188 (2006), no. 1, 107-120. 
[Li07]_ Error analysis of fully discrete mixed finite element schemes for 3-D Maxwell's equations in dispersive media, Comput. Methods Appl. Mech. Engrg. 196 (2007), 30813094 .

[Li11] _ Unified analysis of leap-frog methods for solving time-domain Maxwell's equations in dispersive media, J. Sci. Comput. 47 (2011), 1-26.

[LL08] Q. Lin and J. Li, Superconvergence analysis for Maxwell's equations in dispersive media, Math. Comp. 77 (2008), no. 262, 757-771.

[Lue90] R. Luebbers, A frequency-dependent finite-difference time-domain formulation for dispersive materials, IEEE Trans. Electromag. Compat. 32 (1990), no. 3, 222-227.

[LZC04] T. Lu, P. Zhang, and W. Cai, Discontinuous Galerkin methods for dispersive and lossy Maxwell's equations and PML boundary conditions, IEEE Trans. Geosc. Rem. Sens. 200 (2004), no. 2, 549-580.

[Mon03] P. Monk, Finite elements methods for Maxwell's equations, Oxford Science Publications, 2003.

[MPH06] D. Miklavčič, N. Pavšelj, and F.X. Hart, Electric properties of tissues, Wiley Encyclopedia of Biomedical Engineering, 2006.

[MvMM00] D. Miklavčič, D. Šermov, H. Mekid, and L.M. Mir, A validated model of in vivo electric field distribution in tissues for electrochemotherapy and for DNA electrotransfer for gene therapy, Biochimica et Biophysica Acta 1523 (2000), 73-83.

[Paz83] A. Pazy, Semigroups of linear operators and applications to partial differential equations, Applied Mathematical Sciences, vol. 44, Springer-Verlag, 1983.

$\left[\mathrm{SCL}^{+} 06\right] \quad$ G. Scarella, O. Clatz, S. Lanteri, G. Beaume, S. Oudot, J.-P. Pons, S. Piperno, P. Joly, and J. Wiart, Realistic numerical modelling of human head tissue exposure to electromagnetic waves from cellular phones, Comptes Rendus Physique 7 (2006), no. 5, 501-508.

[Ser05] G. Serša, Application of electroporation in electrochemotherapy of tumors, Electroporation based Technologies and Treatment. International scientific workshop and postgraduate course (Ljubljana, Slovenia), 2005, pp. 42-45.

[SKS ${ }^{+}$92] S.I. Sukharev, V.A. Klenchin, S.M. Serov, L.V. Chernomordik, and Y.A. Chizmadzhev, Electroporation and electrophoretic DNA transfer into cells: the effect of DNA interaction with electropores, Biophys J. 63 (1992), 1320-1327.

[SKVH10] J.D. Schea, P. Kosmas, B.D. Van Veen, and S.C. Hagness, Contrast-enhanced microwave imaging of breast tumors: a computational study using $3 D$ realistic numerical phantoms, Inverse Problems 26 (2010), no. 7.

[Sul92] D.M. Sullivan, A frequency-dependent FDTD method for biological applications, IEEE Trans. Microwave Theory Tech. 40 (1992), no. 3, 532-539.

[Tso91] T.Y. Tsong, Electroporation of cell membranes, Biophys J. 60 (1991), 297-306.

$\left[\mathrm{vCB}^{+} 05\right]$ D. Šel, D. Cukjati, D. Batiuskaite, T. Slivnik, L.M. Mir, and D. Miklavčič, Sequential finite element model of tissue electropermeabilization, IEEE Trans. Biomed. Engrg. 52 (2005), no. $5,816-827$. 
[WSTI09] X. Wei, K. Saito, M. Takahashi, and K. Ito, Performances of an implant cavity slot antenna embedded in the human arm, IEEE Trans. Antennas and Propagat. 57 (2009), no. 4, 894899.

[WXZ10] B. Wang, Z. Xie, and Z. Zhang, Error analysis of a discontinuous Galerkin method for Maxwell's equations in dispersive media, J. Comput. Phys. 229 (2010), no. 22, 8552-8563. 
Centre de recherche INRIA Sophia Antipolis - Méditerranée 2004, route des Lucioles - BP 93 - 06902 Sophia Antipolis Cedex (France)

Centre de recherche INRIA Bordeaux - Sud Ouest : Domaine Universitaire - 351, cours de la Libération - 33405 Talence Cedex Centre de recherche INRIA Grenoble - Rhône-Alpes : 655, avenue de l'Europe - 38334 Montbonnot Saint-Ismier

Centre de recherche INRIA Lille - Nord Europe : Parc Scientifique de la Haute Borne - 40, avenue Halley - 59650 Villeneuve d'Ascq Centre de recherche INRIA Nancy - Grand Est : LORIA, Technopôle de Nancy-Brabois - Campus scientifique 615, rue du Jardin Botanique - BP 101 - 54602 Villers-lès-Nancy Cedex

Centre de recherche INRIA Paris - Rocquencourt : Domaine de Voluceau - Rocquencourt - BP 105 - 78153 Le Chesnay Cedex

Centre de recherche INRIA Rennes - Bretagne Atlantique : IRISA, Campus universitaire de Beaulieu - 35042 Rennes Cedex Centre de recherche INRIA Saclay - Île-de-France : Parc Orsay Université - ZAC des Vignes : 4, rue Jacques Monod - 91893 Orsay Cedex 